\title{
Chemical abundances in the nucleus of the Sagittarius dwarf spheroidal galaxy ${ }^{\star}$
}

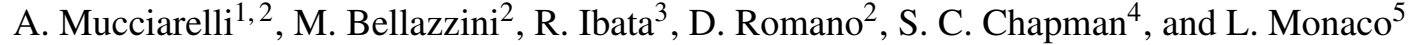 \\ ${ }^{1}$ Dipartimento di Fisica e Astronomia, Università degli Studi di Bologna, via Gobetti 93/2, 40129 Bologna, Italy \\ e-mail: alessio.mucciarelli2@unibo.it \\ 2 INAF - Osservatorio Astronomico di Bologna, via Gobetti 93/3, 40129 Bologna, Italy \\ 3 Observatoire Astronomique de Strasbourg, Université de Strasbourg, CNRS, UMR 7550, 11 rue de l'Université, 67000 Strasbourg, \\ France \\ 4 Department of Physics and Atmospheric Science, Dalhousie University, Halifax, NS B3H 4R2, Canada \\ 5 Departamento de Ciencias Fisicas, Universidad Andres Bello, Fernandez Concha 700, Las Condes, Santiago, Chile
}

Received 27 February 2017 / Accepted 7 May 2017

\begin{abstract}
We present iron, magnesium, calcium, and titanium abundances for 235 stars in the central region of the Sagittarius dwarf spheroidal galaxy (within $9.0^{\prime} \simeq 70 \mathrm{pc}$ from the centre) from medium-resolution Keck/Deep Imaging Multi-Object Spectrograph spectra. All the considered stars belong to the massive globular cluster M 54 or to the central nucleus of the galaxy (Sgr, N). In particular we provide abundances for 109 stars with $[\mathrm{Fe} / \mathrm{H}] \geq-1.0$, more than doubling the available sample of spectroscopic metallicity and $\alpha$ elements abundance estimates for Sgr dSph stars in this metallicity regime. We find for the first time a metallicity gradient in the Sgr, $\mathrm{N}$ population, whose peak iron abundance goes from $[\mathrm{Fe} / \mathrm{H}]=-0.38$ for $R \leq 2.5^{\prime}$ to $[\mathrm{Fe} / \mathrm{H}]=-0.57$ for $5.0<R \leq 9.0^{\prime}$. On the other hand, the trends of $[\mathrm{Mg} / \mathrm{Fe}],[\mathrm{Ca} / \mathrm{Fe}]$, and $[\mathrm{Ti} / \mathrm{Fe}]$ with $[\mathrm{Fe} / \mathrm{H}]$ are the same over the entire region explored by our study. We reproduce the observed chemical patterns of the Sagittarius dwarf spheroidal as a whole with a chemical evolution model which implies that a high mass progenitor $\left(M_{D M}=6 \times 10^{10} M_{\odot}\right)$ and a significant event of mass-stripping occurred a few Gyr ago, presumably starting at the first peri-Galactic passage after infall.
\end{abstract}

Key words. galaxies: dwarf - Local Group - galaxies: stellar content - stars: abundances

\section{Introduction}

The Sagittarius dwarf spheroidal galaxy (Sgr dSph, Ibata et al. $1994)$ is the most emblematic case of a Galactic satellite that is being disrupted by the tidal field of the Milky Way. The main body of the system is a large (half-light radius $r_{\mathrm{h}}=$ $2.6 \mathrm{kpc}$, Majewski et al. 2003) low-surface brightness gas-less elongated spheroid. It is located behind the Galactic bulge, as seen from the Sun, and $\sim 6.5 \mathrm{kpc}$ below the Galactic plane, lying at a distance of $D=26.3 \pm 1.8 \mathrm{kpc}$ from us (Monaco et al. 2004). On the other hand, the two arms of its tidal stream are traced all over the sky, out to $D \simeq 100 \mathrm{kpc}$ (see Majewski et al. 2003; Belokurov et al. 2014, and references therein). The process of disruption of Sgr dSph contributes to the build-up of the Galactic halo in terms of dark matter, stars, and globular clusters (see e.g. Majewski et al. 2003; Bellazzini et al. 2003; Monaco et al. 2003; Law \& Majewski 2010; Gibbons et al. 2017; de Boer et al. 2014).

According to the compilation by McConnachie (2012), the present-day stellar mass and dynamical mass (within $r_{\mathrm{h}}$ ) of the main body are $M_{\star} \sim 2 \times 10^{7} M_{\odot}$ (Ibata et al. 2004) and $M_{\text {dyn }} \sim 2 \times 10^{8} M_{\odot}$ (Grcevish \& Putman 2009), respectively, but there is a consensus that the mass of the progenitor must have been a factor of ten larger (Niederste-Ostholt et al. 2012; de Boer et al. 2014; Gibbons et al. 2017). The stellar population of the main body is dominated by an intermediateage (mean age $\gtrsim 5 \mathrm{Gyr}$ ) relatively metal-rich $(\langle[\mathrm{Fe} / \mathrm{H}]\rangle \simeq-0.5$ )

* Full Table 1 is only available at the CDS via anonymous ftp to cdsarc.u-strasbg.fr (130.79.128.5) or via

http://cdsarc.u-strasbg.fr/viz-bin/qcat?]/A+A/605/A46 population (Bellazzini et al. 2006b; Siegel et al. 2007, and references therein), with an old and metal-poor component contributing $~ 10$ per cent to the stellar budget (Monaco et al. 2003; Hamanowicz et al. 2016).

In spite of being extended over several kpc, Sgr dSph hosts an over-density of stars on the $\$ 100 \mathrm{pc}$ scale lying straight at its centre (see Fig. 1), with all the characteristics of the stellar nuclei very frequently found in dwarf ellipticals as well as in galaxies of other morphological types (see Monaco et al. 2005a; Bellazzini et al. 2008, B08 hereafter, references and discussion therein). The massive and metal-poor $([\mathrm{Fe} / \mathrm{H}]=-1.56$, Carretta et al. 2010b) globular cluster M 54 (NGC 6715) coincides with this nucleus (Sgr, $\mathrm{N}$ hereafter) in position and radial velocity. B08 showed that the two systems have different surface density profiles and, above all, very different velocity dispersion profiles, supporting the idea that M 54 formed independently from the metal-rich nucleus and was brought to its current position by dynamical friction (see also Ibata et al. 2009). The viability of the scenario was demonstrated both analytically (Monaco et al. 2005a) and with $N$-body simulations (B08), and it found independent support from chemical tagging arguments (Carretta et al. 2010a).

The chemical composition of stars in Sgr dSph is of great interest and indeed several spectroscopic studies addressed this problem, for example Smecker-Hane \& McWilliam (2002, SM02 hereafter), Bonifacio et al. (2000, 2004), Monaco et al. (2005b, M05 hereafter), Sbordone et al. (2007, S07 hereafter), that re-analysed also Bonifacio et al.'s samples, Chou et al. (2007), Carretta et al. (2010a,b, C10a and C10b, respectively hereafter), and McWilliam et al. (2013, MWM13 hereafter). In 
particular, MWM13 present a comprehensive view of all the iron and $\alpha$-element abundances available in the literature for the main body of the galaxy. Surprisingly enough they amount to less than 70 stars, the large majority having $[\mathrm{Fe} / \mathrm{H}] \geq-1.0$. Hence we are far from having a satisfactory sampling of the metallicity distribution and abundance patterns in this crucial system.

To make a significant step ahead in this direction, we present here the elemental abundances of iron, magnesium, calcium, and titanium for a large and homogeneous subsample of the M 54 and Sgr, N stars studied in B08 using the Keck/Deep Imaging Multi-Object Spectrograph (DEIMOS) spectra. These authors estimated the metallicity of their stars using the calcium triplet technique, with the main purpose of classifying stars for the analysis of the kinematics of the system, which was the final goal of their study. Conversely, here we analyse the chemical abundances of stars derived via synthetic spectroscopy on individual atomic transition lines. Since the main abundance patterns and trends in M 54 have been already studied and discussed in detail by $\mathrm{C} 10 \mathrm{~b}$, we focus our analysis on the Sgr population.

The plan of the paper is the following. In Sect. 2 we describe the sample and the data analysis and in Sect. 3 we compare our results with those available in the literature. Section 4 is devoted to the analysis of the observed abundance distributions and chemical patterns, also by means of chemical evolution models. The main results of the analysis are summarised and discussed in Sect. 5.

\section{Data and abundance measures}

\subsection{Observational dataset}

The spectroscopic data analysed in this work are part of the dataset already discussed in B08. The bulk of the B08 sample was constituted by Keck/DEIMOS spectra at $R=\frac{\lambda}{\Delta \lambda} \simeq 6600$, fully analogous to those used by Mucciarelli et al. (2012) to derive abundances of a few chemical elements in the globular cluster NGC 2419 with spectral synthesis of selected atomic lines. The original sample of B08 used both slits and holes DEIMOS spectra. In the present work, we limit the analysis to the DEIMOS slits spectra because the DEIMOS holes spectra cover a smaller spectral range that prevents the measurement of the $\mathrm{Mg}$ abundances and leads to a significant decrease in the number of available Fe lines (down to only five instead of $\sim 15$ ). We perform the same analysis as in Mucciarelli et al. (2012) on 235 stars observed with Keck/DEIMOS, whose membership to M 54 or Sgr, N has been established by B08 and selected to have signal-to-noise ratio ( $\mathrm{S} / \mathrm{N}$ ) larger than $\sim 20-30$ (up to $\sim 120-130$ ) in the spectral region $\sim 8400-8700 \AA$. This sample is confined to the nuclear region of Sgr. All the stars are within $9.0^{\prime} \simeq 70 \mathrm{pc}$ from the centre of the system and they are all enclosed within the innermost density contour of Fig. 1. The position on the colourmagnitude diagram (CMD) of the spectroscopic targets analysed in this paper is shown in Fig. 2. We limited the analysis to this subsample of the B08 data to keep the observational material and the analysis as homogeneous as possible, to have the most self-consistent scale of relative abundances.

The original B08 sample was selected to minimise the contamination by Galactic foreground stars by choosing targets lying close to the main branches of M 54 and Sgr, N on the CMD. This implies that the resulting metallicity distribution for Sgr, N is biased against metal-poor stars not only because in this central region it is overwhelmed by M54 stars but also because stars with intermediate colours between the blue red giant branch (RGB) of M 54 and the main red RGB of Sgr were not included

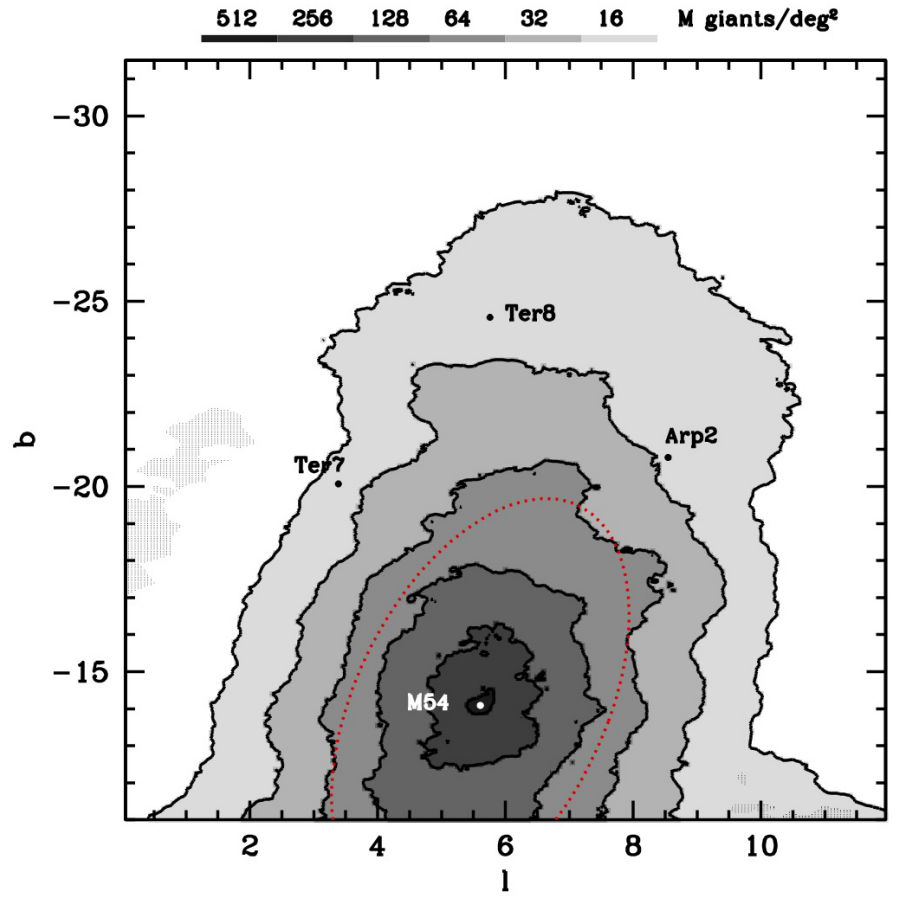

Fig. 1. Surface density map of the southern half of the main body of Sgr dSph as traced by M giants from 2MASS (Skrutskie et al. 2006), selected according to Majewski et al. (2003). The light-shaded areas have $E(B-V)>0.3$ according to the extinction maps by Schlegel et al. (1998). The globular clusters associated to Sgr dSph are marked and labelled. The dashed ellipse is the half-light contour of the best-fit King (1962) model by Majewski et al. (2003); the half-light radius along the major axis is taken from McConnachie (2012). For $b \geq-13.0^{\circ}$ the contamination by foreground Galactic M-giants and the high extinction prevent a reliable tracing of the Sgr density, affecting the shape of the outer iso-density contours. At the distance of $\operatorname{Sgr} \mathrm{dSph}(D=26.3 \mathrm{kpc}$, Monaco et al. 2004), one degree corresponds to $459 \mathrm{pc}$.

in the sample ${ }^{1}$. On the other hand, our sample is fully adequate to study the peak of the metal-rich population and to trace the trends of abundance ratios with $[\mathrm{Fe} / \mathrm{H}]$.

\subsection{Atmospheric parameters}

Effective temperatures $\left(T_{\text {eff }}\right)$ and surface gravities $(\log g)$ have been derived from the photometric catalogue by Monaco et al. (2002). In particular, $T_{\text {eff }}$ have been obtained from the ( $V-$ $I)_{0}-T_{\text {eff }}$ calibration provided by Alonso et al. (1999) and by adopting the colour excess $E(B-V)=0.14 \pm 0.03 \mathrm{mag}$ (Layden \& Sarajedini 2000). Because the Alonso et al. (1999) calibration used the Johnson I-filter, the Cousin I-band magnitudes by Monaco et al. (2004) have been transformed into the Johnson photometric system adopting the transformation provided by Bessell (1979). Internal uncertainties in $T_{\text {eff }}$ are estimated including errors in photometric data and reddening which are of the order of $\sim 60-100 \mathrm{~K}$.

Surface gravities have been calculated through the StefanBoltzmann relation and by adopting the photometric $T_{\text {eff }}$ described above, the bolometric corrections by Alonso et al. (1999), a stellar mass of $0.8 M_{\odot}$, and a true distance modulus

\footnotetext{
1 We note that any attempt to obtain an unbiased metallicity distribution in Sgr would require us to sample a region far from M 54 or other Sgr globulars and would imply taking the spectra of a large number of Galactic foreground stars having colour and magnitude in the range spanned by Sgr red giants.
} 


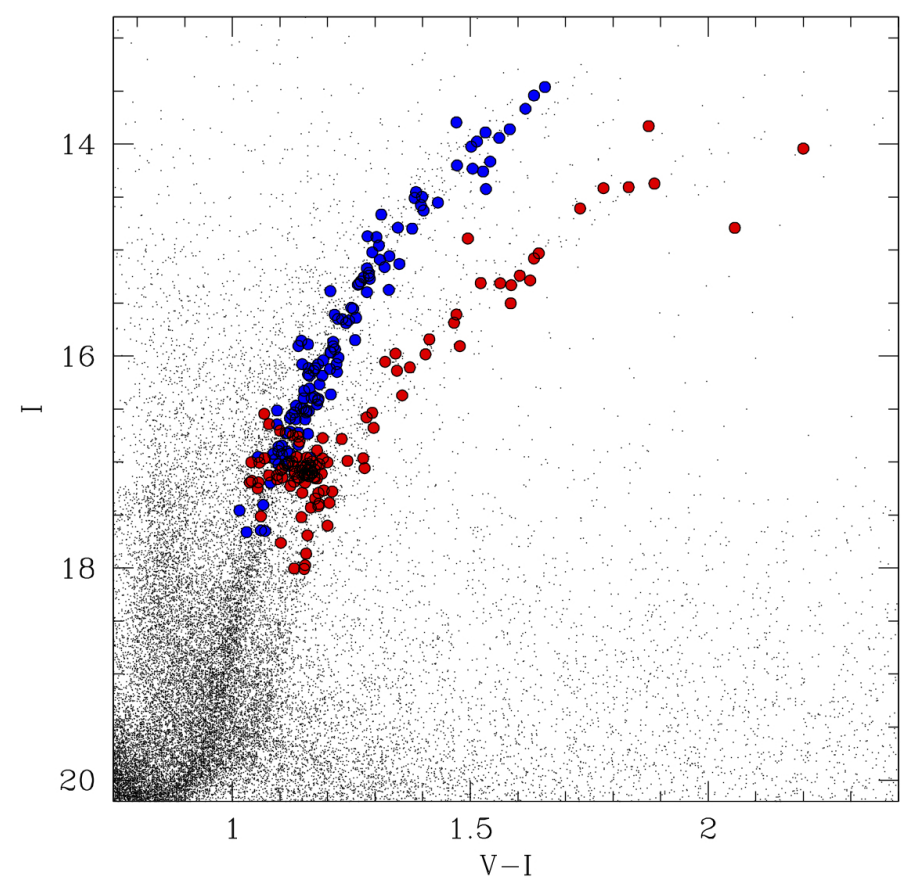

Fig. 2. Colour-magnitude diagram of the inner $9.0^{\prime}$ of M54 (Monaco et al. 2002) with superimposed the position of the Keck/DEIMOS spectroscopic targets: red circles are the stars with $[\mathrm{Fe} / \mathrm{H}]>-1.0$ dex, blue circles those with $[\mathrm{Fe} / \mathrm{H}]<-1.0$ dex.

$(m-M)_{0}=17.10 \pm 0.15 \mathrm{mag}$ (Monaco et al. 2004). Propagating the uncertainties in $T_{\mathrm{eff}}, E(B-V)$, mass, and distance modulus we obtain uncertainties in $\log g$ of \pm 0.1 .

Microturbulent velocities $\left(v_{\mathrm{t}}\right)$ cannot be derived directly from the spectra, as is usually done with high-resolution spectra, because the spectral resolution of the Keck/DEIMOS spectra prevents the measurement of weak Fe I lines. For this work, the $\log g-v_{\mathrm{t}}$ calibration provided by Kirby et al. (2009) has been adopted. We note that the formal errors in $\log g$ lead to uncertainties in $v_{\mathrm{t}}$ of only $\pm 0.02-0.03 \mathrm{~km} \mathrm{~s}^{-1}$. Because the used lines are strong enough to be sensitive to the velocity fields, we assumed a conservative uncertainty of $0.2 \mathrm{~km} \mathrm{~s}^{-1}$.

\subsection{Abundance analysis}

Chemical abundances have been derived with our own code SALVADOR through a $\chi^{2}$-minimization between the observed line and a grid of synthetic spectra calculated with the appropriate atmospheric parameters and varying only the abundance of the species under scrutiny. The synthetic spectra have been calculated using the code SYNTHE $^{2}$ and including all the transitions included in the Kurucz/Castelli linelists. Model atmospheres have been calculated with the last version of the ATLAS9 code $^{3}$. The transitions to be used for the chemical analysis have been selected according to suitable synthetic spectra calculated over the entire wavelength range and convolved with a Gaussian profile to reproduce the spectral resolution of the Keck/DEIMOS spectra. We picked only unblended transitions using the atomic data listed in the Kurucz/Castelli linelists ${ }^{4}$. We included in our linelist $15 \mathrm{Fe}$ I lines, four Ti I lines, the Mg I line at $8806.7 \AA$, and the

\footnotetext{
2 http://wwwuser.oats. inaf.it/castelli/sources/synthe. html

3 http://wwwuser.oats.inaf.it/castelli/sources/ atlas9codes.html

4 http://wwwuser.oats. inaf.it/castelli/linelists.html
}

two strongest CaT lines at 8552 and $8662 \AA$. For each transition (except the Ca II lines) the $\chi^{2}$-minimization has been performed within a fitting-window of $\sim 4-5 \AA$. Only for the Ca II lines did we adopt a different strategy: following Mucciarelli et al. (2012), the $\mathrm{Ca}$ abundance has been derived by fitting only the wings of these lines and disregarding the core. In fact, the core of the $\mathrm{Ca}$ II lines forms in the outermost photospheric layers and it is sensitive to the inadequacies of the 1-dimensional model atmospheres and it suffers from severe departures from local thermodynamical equilibrium.

All the observed spectra have been previously normalised by fitting the entire spectrum with a cubic spline function. The continuum location has been refined by using pre-selected spectral windows representative of the continuum (i.e. without spectral features): a cubic spline has been calculated considering the median values of the continuum windows and then used to normalise, in a self-consistent way, both synthetic and observed spectra.

Because of the large range of metallicity covered by the entire sample (including both metal-poor M 54 stars and metal-rich Sgr, N stars), a unique list of continuum windows cannot be suitable for all the stars. The entire analysis procedure has been iteratively performed: a first analysis has been done adopting a preliminary set of windows and then refined with an appropriate list of continuum windows selected according to the stellar metallicity.

The procedure of normalisation can be critical at the DEIMOS spectral resolution, especially for the most metal-rich stars, where the blanketing conditions can make difficult an appropriate location of the continuum level. In order to check the stability of our abundances against the method to normalise the spectra, we repeat the analysis for a subsample of spectra adopting the approach originally described by Shetrone et al. (2009) and already implemented in SALVADOR (see Mucciarelli et al. 2012). In this approach, the observed spectrum is divided by the spline obtained by fitting the residuals between the observed spectrum and the best-fit synthetic spectrum obtained in a first iteration. The procedure is repeated if the derived abundance changes significantly. We analysed some tens of stars picked in order to sample different metallicity and S/N. For bright, metalpoor stars the absolute differences in the derived abundances between the two methods are smaller than 0.02 dex, while for the bright, metal-rich stars they do not exceed 0.04 dex. For faint stars, with low $\mathrm{S} / \mathrm{N}$, the differences between the two approaches remain smaller than 0.1 dex. However, these abundance differences are, on average, compatible with zero, pointing out that the two approaches are substantially equivalent. The use of the method of Shetrone et al. (2009) does not change significantly the derived metallicity distribution of Sgr, $\mathrm{N}$ and our conclusions.

Uncertainties in the chemical abundances have been estimated by taking into account two main sources of error:

1. Uncertainties arising from the fitting procedure that have been estimated by using Montecarlo simulations. For each spectral line, 300 simulated spectra have been created by resampling the best-fit synthetic spectrum at the pixel-scale of the observed spectrum and then by adding Poissonian noise in order to mimic the measured $\mathrm{S} / \mathrm{N}$ per pixel. This set of simulated noisy spectra has been re-analysed with the same procedure used for real spectral features and the standard deviation of the derived abundance distribution is taken as the uncertainty. For the lines observed in the brightest stars $(I \simeq 14.0$ and $S / N \sim 130)$, the fitting uncertainty is about 
$\pm 0.03-0.04$ dex, increasing up to $\sim 0.2$ dex for the faintest stars $(I \simeq 18.0$ and $S / N \sim 20-30)$. Only for the $\mathrm{Ca}$ lines is the error associated with the photon noise smaller (reaching \pm 0.05 dex for the faintest stars) because of the large number of pixels used in the fitting procedure.

2. Uncertainties arising from the atmospheric parameters that have been estimated by repeating the analysis varying each time one atmospheric parameter by the corresponding uncertainty. The used lines are mainly sensitive to $T_{\text {eff }}$ and $v_{\mathrm{t}}$, while marginally sensitive to $\log g$. A variation in $\log g$ of \pm 0.1 leads to a variation in $[\mathrm{Fe} / \mathrm{H}]$ of $\pm 0.02-0.03$ dex and virtually a null variation in the other abundance ratios. On the other hand, a variation in $T_{\text {eff }}$ of $\pm 100 \mathrm{~K}$ leads to typical changes of $\pm 0.05-0.07$ dex in $[\mathrm{Fe} / \mathrm{H}], \pm 0.10 \mathrm{dex}$ in $[\mathrm{Mg} / \mathrm{Fe}]$, $\mp 0.05 \mathrm{dex}$ in $[\mathrm{Ca} / \mathrm{Fe}]$, and up to $\pm 0.15-0.20 \mathrm{dex}$ in $[\mathrm{Ti} / \mathrm{Fe}]$ (as the $\mathrm{Ti} \mathrm{I}$ lines used in this work are very sensitive to changes in $T_{\text {eff }}$ ). Finally, a variation of $\pm 0.2 \mathrm{~km} \mathrm{~s}^{-1}$ in $v_{\mathrm{t}}$ leads to a change of $\pm 0.10-0.15 \mathrm{dex}$ in $[\mathrm{Fe} / \mathrm{H}]$ and smaller than $0.1 \mathrm{dex}$ for the other abundance ratios.

Finally, we derived abundances of $\mathrm{Fe}, \mathrm{Mg}, \mathrm{Ca}$, and Ti for 235 giant stars in M 54 and Sgr, N. This is by far the largest sample of chemical abundances in the main body of Sgr dSph ever presented. While a large fraction of the sample is attributable to M 54 (i.e. the vast majority of the stars with $-1.8 \lesssim[\mathrm{Fe} / \mathrm{H}] \lessgtr$ -1.2 ), there are also 109 stars having $[\mathrm{Fe} / \mathrm{H}] \geq-1.0$ (hence unambiguously associated with Sgr, N), 89 of which were never analysed before in terms of chemical composition. Hence this study more than doubles the number of stars with elemental abundances in the main metal-rich stellar component of Sgr dSph. In Fig. 2 the targets are plotted in the CMD according to their metallicity, red circles for the stars with $[\mathrm{Fe} / \mathrm{H}] \geq$ -1.0 dex (associated to Sgr, N) and blue circles for those with $[\mathrm{Fe} / \mathrm{H}]<-1.0$ (mainly stars belonging to M 54). This figure is an analogue to Fig. 10 in B08, confirming that the metal-rich stars are all associated to the reddest RGB visible in the CMD and corresponding to Sgr, N. It is important to note that the metalrich sample is largely composed of red clump (RC) stars, while the metal-poor one is made up only of RGB stars.

\section{Comparison with previous studies}

In Fig. 3 we show that the new $[\mathrm{Fe} / \mathrm{H}]$ estimates based on synthetic spectral analysis are in agreement with those by B08 from the calcium triplet, within the uncertainties. A small systematic deviation is apparent in the most metal-rich regime, where it is known that the CaT technique may loose some sensitivity and reliability (see e.g. the discussion in Carrera et al. 2007).

We note that one of the four Sgr, N members found by B08 to have $[\mathrm{Fe} / \mathrm{H}]_{\mathrm{CaT}} \leq-2.0$ is included in the present sample (namely \# 3801005) and is confirmed to have an iron abundance less than $\frac{1}{100}$ solar by spectral analysis of iron lines $([\mathrm{Fe} / \mathrm{H}]=-2.18 \pm 0.20)$. Figure 4 shows the comparison between the spectrum of the star \# 3801005 and the star \# 3801481, with similar atmospheric parameters and a metallicity of $[\mathrm{Fe} / \mathrm{H}]=-1.58 \mathrm{dex}$, compatible with the bulk of the M54 metallicity distribution. The difference in the metallicity of the two targets is clearly visible in the strengths of both the Fe I and Ca II lines shown in Fig. 4. The good match with the CaT metallicity of B08 suggests that also the other four candidates found in that study are likely to be genuine very metal-poor stars. We stress that very few stars with metallicity $[\mathrm{Fe} / \mathrm{H}] \leq-1.5$, as derived from direct spectroscopic measures, are known in the main body of Sgr (see e.g. MWM13 and Fig. 4 in Sbordone et al. 2015).

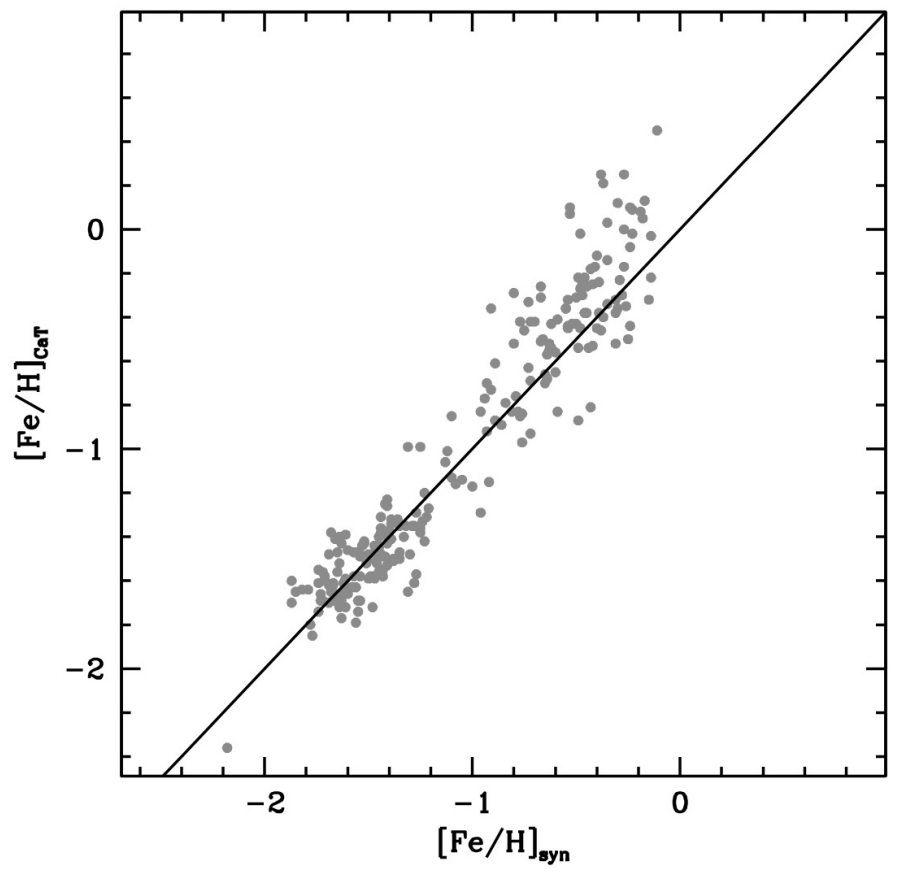

Fig. 3. Comparison of iron abundances obtained by spectral synthesis of iron lines in the present analysis and those derived by B08 with the calcium triplet technique, from the same spectra. The diagonal line has slope $=1.0$.

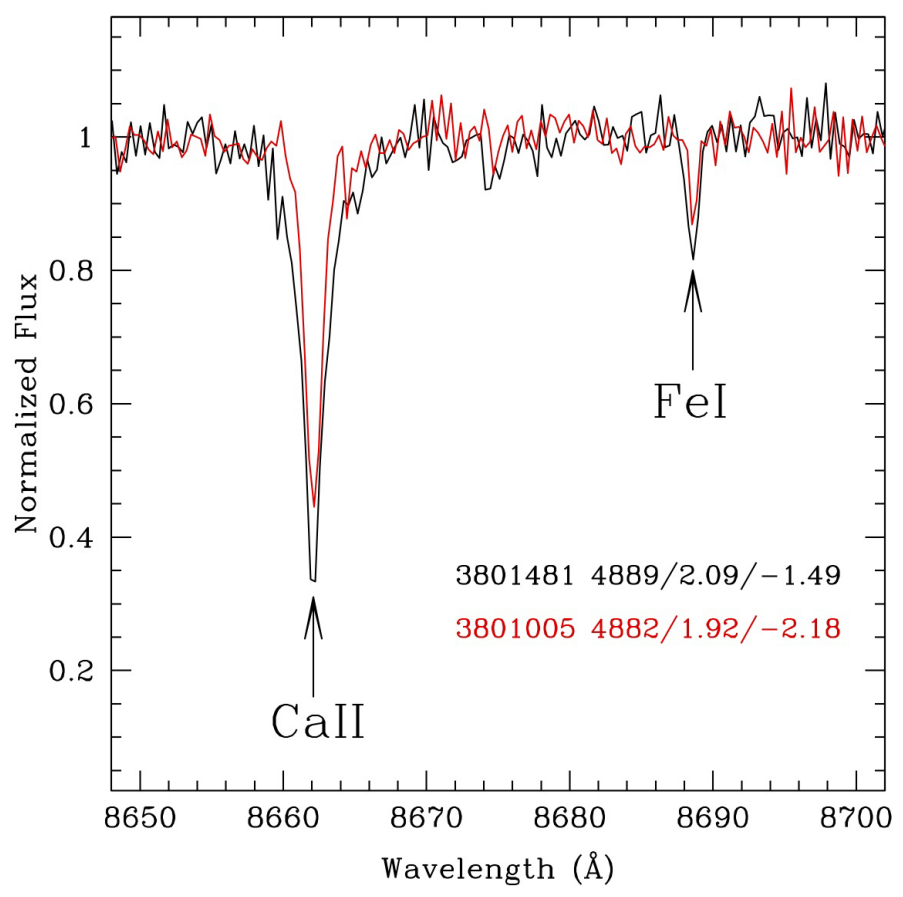

Fig. 4. Comparison between the spectra of the stars \# 3801005 (red line) and \# 3801481 (black line) around the Ca II line at $8662 \AA$ and the Fe I line at $8689 \AA$. The two stars have similar atmospheric parameters $\left(T_{\text {eff }}=4882 \mathrm{~K}\right.$ and $\log g=1.92$ for \# 3801005 and $T_{\text {eff }}=4889 \mathrm{~K}$ and $\log g=2.09$ for \# 3801481) but different iron abundances.

In Fig. 5 we plot the difference in abundance ratios between our estimates and those previously available in the literature for stars in common. It is pretty clear that a fully meaningful comparison can be performed only with the $\mathrm{C} 10 \mathrm{~b}$ sample, with which we have sixteen stars in common (only one with $[\mathrm{Fe} / \mathrm{H}]>-1.0$, 


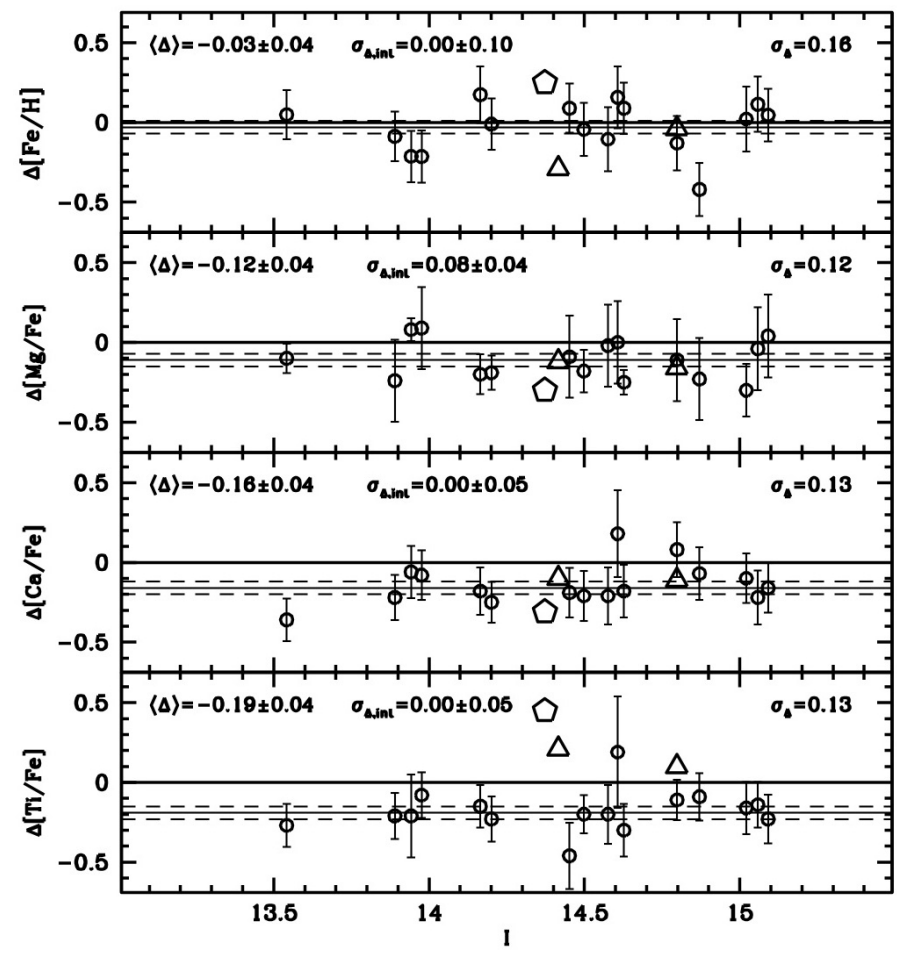

Fig. 5. Differences in the abundance of stars in common between this work and other analyses ( $\Delta=$ other analyses - this work). The small empty circles are from $\mathrm{C} 10 \mathrm{~b}$, empty triangles from M05, empty pentagons from MWM13. The thin continuous line is the mean of the differenc with respect to $\mathrm{C} 10 \mathrm{~b}$ and the thin dashed lines enclose the errors about the mean, as derived with the Maximum Likelihood algorithm (value reported in the upper left corner of each panel, together with the intrinsic dispersion and the associated uncertainties). The value of the straight standard deviation of the difference is also reported in the upper right corner of each panel.

however), while two are in common with M05 and one in common with MWM13 $3^{5}$. For this reason, and since the $\mathrm{C} 10 \mathrm{~b}$ analysis is based on higher resolution spectra, we take it as a reference for comparison. The overall comparison is satisfactory.

We compute the mean and intrinsic standard deviation $\left(\sigma_{\text {int }}\right)$ of the differences between the abundance ratios by $\mathrm{C} 10 \mathrm{~b}$ and our own ones using the Maximum Likelihood (ML) algorithm described in Mucciarelli et al. (2012). In all the cases $\sigma_{\text {int }}$ is consistent with zero, within the uncertainties. Indeed, the overall standard deviation reported in the upper right corner of each panel is always of the order of the uncertainties on individual abundance estimates.

The mean differences in the abundance ratios, with the associated uncertainties, are reported in the upper left corner of each panel of Fig. 5. While the mean difference in $[\mathrm{Fe} / \mathrm{H}]$ is null, within the uncertainties, there are small but significant differences in the zero point of abundance ratios involving the $\alpha$ elements. We note that for $[\mathrm{Mg} / \mathrm{Fe}]$ and $[\mathrm{Ca} / \mathrm{Fe}]$ the three points from M05 and MWM13 are consistent with the distribution of $\mathrm{C} 10 \mathrm{~b}$ points. This is not the case for $[\mathrm{Ti} / \mathrm{Fe}]$; we will see below that this ratio is indeed more problematic (see also MWM13 for a discussion). To be compliant with $\mathrm{C} 10 \mathrm{~b}$, we apply to all our abundance ratios the mean shifts reported in Fig. 5. The

\footnotetext{
5 For abundances derived from one single line, $\mathrm{C} 10 \mathrm{~b}$ does not provide uncertainties. We exclude these values from this and following comparisons. The same is true for one of the stars in the sample by Chou et al. (2007).
}

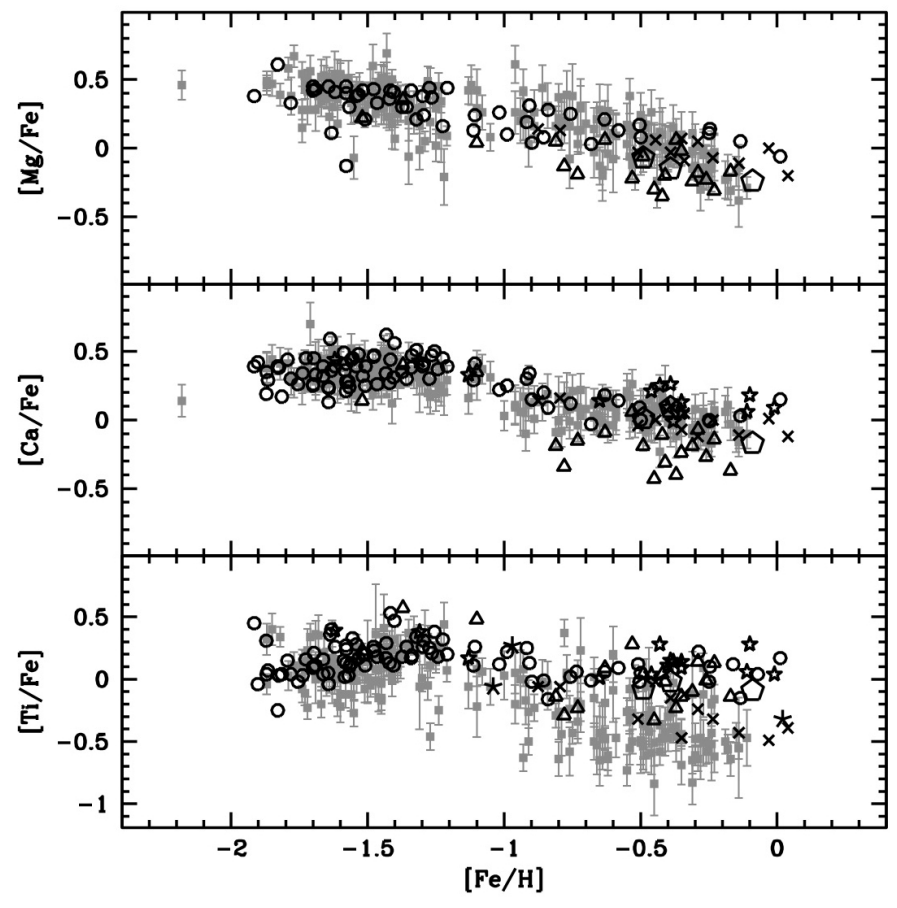

Fig. 6. Comparison of the trends of the $\alpha$-elements considered here versus $[\mathrm{Fe} / \mathrm{H}]$ obtained from the present analysis (small grey squares) with datasets from the literature. Small empty circles: C10b; $\times$ symbols: S07; empty stars: SM02; empty triangles: M05; empty pentagons: MWM13; asteriks: Chou et al. (2007).

estimates used in the following and listed in Table 1 have been corrected accordingly.

In Fig. 6 we compare our results with all the abundances for stars in the main body of Sgr available in the literature in the planes opposing $[\mathrm{Mg} / \mathrm{Fe}],[\mathrm{Ca} / \mathrm{Fe}]$, and $[\mathrm{Ti} / \mathrm{Fe}]$ to $[\mathrm{Fe} / \mathrm{H}]$. The overall agreement is good for $[\mathrm{Mg} / \mathrm{Fe}]$ and $[\mathrm{Ca} / \mathrm{Fe}]$, especially if the variety of sources is considered. The agreement is satisfactory also for $[\mathrm{Ti} / \mathrm{Fe}]$ up to $[\mathrm{Fe} / \mathrm{H}] \simeq-0.8$. However, at larger metallicity the spread between different sets of measures is large and probably dominated by systematics. In particular, the bulk of our estimates is significantly lower than the $[\mathrm{Ti} / \mathrm{Fe}]$ range spanned by most of the literature values. However, we note that a significant scatter does exist also among the different $[\mathrm{Ti} / \mathrm{Fe}]$ trends with $[\mathrm{Fe} / \mathrm{H}]$ found by other authors, in the metal-rich stars of Sgr, N. The origin of the discrepancy is not clear: the Ti lines are very sensitive to temperature and microturbulent velocity and we cannot rule out that systematic uncertainties in the $T_{\text {eff }}$ scale for the metal-rich stars may lead to systematic mis-estimates of the Ti abundance (see also MWM13). Due to these uncertainties in the analysis and to the uncertainties in the Ti yields that affect the chemical evolution models (see Sect. 4.2), we exclude this element from the following discussion.

From the comparisons above, we conclude that our $\mathrm{Fe}, \mathrm{Mg}$, and $\mathrm{Ca}$ abundances are reliable over the whole range of metallicity spanned by our data, while $[\mathrm{Ti} / \mathrm{Fe}]$ values in the metal-rich regime must be considered with great caution. In the following we will limit our analysis to our own sample to take advantage of the highest degree of internal homogeneity and consistency of our measurements. This is an important step forward in our view of the chemical patterns in Sgr dSph, since the mutual consistency of the abundance sets from various authors is not well established; systematic differences have been noted (see e.g. MWM13) and can be seen also in Fig. 6. 
Table 1. Atmospheric parameters and abundances.

\begin{tabular}{|c|c|c|c|c|c|c|c|c|c|c|c|c|c|}
\hline ID & $\begin{array}{c}\mathrm{RA}_{J 2000} \\
\text { [deg] }\end{array}$ & $\begin{array}{c}\operatorname{Dec}_{J 2000} \\
\text { [deg] }\end{array}$ & $\begin{array}{l}T_{\text {eff }} \\
{[\mathrm{K}]}\end{array}$ & $\begin{array}{c}\log g \\
{\left[\mathrm{~cm} \mathrm{~s}^{-2}\right]}\end{array}$ & $\begin{array}{c}v_{\mathrm{t}} \\
{\left[\mathrm{km} \mathrm{s}^{-1}\right]}\end{array}$ & {$[\mathrm{Fe} / \mathrm{H}]$} & err & {$[\mathrm{Mg} / \mathrm{Fe}]$} & err & $\overline{[\mathrm{Ca} / \mathrm{Fe}]}$ & err & {$[\mathrm{Ti} / \mathrm{Fe}]$} & err \\
\hline 3700223 & 283.655137 & -30.398678 & 4581 & 1.47 & 1.8 & -1.47 & 0.14 & 0.40 & 0.08 & 0.33 & 0.12 & 0.08 & 0.08 \\
\hline 3700626 & 283.657212 & -30.400988 & 4804 & 2.02 & 1.7 & -1.44 & 0.16 & 0.39 & 0.12 & 0.44 & 0.13 & 0.25 & 0.11 \\
\hline 3800223 & 283.813879 & -30.403892 & 4060 & 0.60 & 2.0 & -1.21 & 0.14 & 0.09 & 0.07 & 0.29 & 0.11 & 0.07 & 0.09 \\
\hline 3800306 & 283.819591 & -30.464056 & 4586 & 1.32 & 1.8 & -1.28 & 0.14 & 0.44 & 0.07 & 0.20 & 0.11 & -0.05 & 0.12 \\
\hline 3800422 & 283.781041 & -30.463381 & 4460 & 1.38 & 1.8 & -1.39 & 0.14 & 0.25 & 0.08 & 0.36 & 0.11 & 0.16 & 0.11 \\
\hline 3800471 & 283.718195 & -30.441672 & 4498 & 1.36 & 1.8 & -1.57 & 0.15 & 0.41 & 0.08 & 0.33 & 0.13 & 0.06 & 0.09 \\
\hline 3800508 & 283.725104 & -30.440299 & 4614 & 1.51 & 1.8 & -1.68 & 0.15 & 0.38 & 0.08 & 0.38 & 0.11 & 0.07 & 0.08 \\
\hline
\end{tabular}

Notes. The full table is available at the CDS.

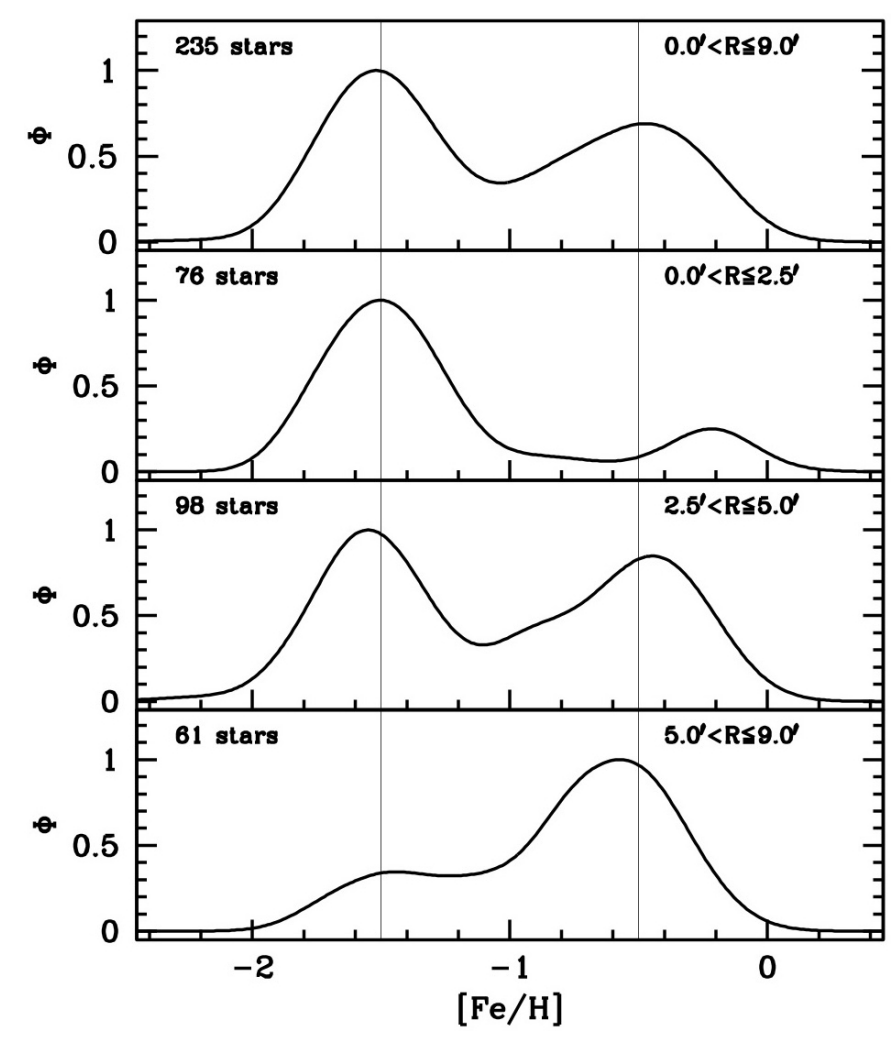

Fig. 7. Metallicity distribution of stars in our sample in different radia annuli around the centre of M 54/Sgr, N. The distributions are in the form of generalised histograms normalised at their maximum. The thin vertical lines are for reference and are placed at $[\mathrm{Fe} / \mathrm{H}]=-1.50$ and $[\mathrm{Fe} / \mathrm{H}]=-0.5$, near the mean of the two peaks, corresponding to M 54 and Sgr, N.

\section{Chemical patterns in Sgr, $\mathrm{N}$}

In Fig. 7 we show the metallicity distribution of our sample in different radial annuli. We recall again that this distribution is biased by the inclusion of M 54 and by the strong selection toward stars lying near the main $\mathrm{RGB} / \mathrm{RC}$ sequences in the CMD, that is toward stars belonging to M 54 or to the main metal-rich population of Sgr. Indeed these populations are well traced by the two strong peaks that characterise the total distribution in the upper panel of Fig. 7. Still, moving to more external annuli, with the progressive decreasing of the M 54 peak, the metal-poor tail of the Sgr, N population becomes discernible as a left wing asymmetry of the metal-rich peak.

Using the ML algorithm on various subsamples, we tried to estimate the mean metallicity of the populations corresponding to the main peaks and to put constraints on their intrinsic dispersion. Attempting to isolate the purest sample of bona-fide M 54 members, we selected 61 stars having $-2.0<[\mathrm{Fe} / \mathrm{H}]<-1.0$ and $R \leq 2.5^{\prime}$. From this subsample we obtain $\langle[\mathrm{Fe} / \mathrm{H}]\rangle=-1.52 \pm$ 0.02 and $\sigma_{\text {int }}=0.09 \pm 0.03$, in good agreement with C10b. For the overall sample of the $\mathrm{Sgr}, \mathrm{N}$ metal-rich component, that is the 109 stars with $[\mathrm{Fe} / \mathrm{H}] \geq-1.0$, we find $\langle[\mathrm{Fe} / \mathrm{H}]\rangle=-0.52 \pm 0.02$ and $\sigma_{\text {int }}=0.17 \pm 0.02$. Even with the cut at $[\mathrm{Fe} / \mathrm{H}]=-1.0$, the metallicity distribution of Sgr, $\mathrm{N}$ is much broader than that of M 54. We note that the modes of the distribution shown in the upper panel of Fig. 7 occur at $[\mathrm{Fe} / \mathrm{H}]=-1.52$ and $[\mathrm{Fe} / \mathrm{H}]=-0.47 \mathrm{dex}$, hence the ML means above trace pretty well the position of the two metallicity peaks.

In recent years several studies have addressed the metallicity distribution of stars in the Sagittarius stream, with different techniques. All of them found a metallicity gradient along the Stream (see e.g. Bellazzini et al. 2006a; Monaco et al. 2007; Chou et al. 2007; Keller et al. 2010; Hyde et al. 2015; Gibbons et al. 2017, and references therein) hinting at a pre-existing metallicity gradient in the population of the progenitor. Indeed, photometric evidence of a metallicity gradient within the main body was already presented by Bellazzini et al. (1999) and Alard (2001). Here we present the first spectroscopic evidence for a metallicity gradient in the main body of Sgr dSph, since Fig. 7 shows that the peak of the metal-rich population changes with projected distance from the centre within the stellar nucleus of Sgr. This peak is at $[\mathrm{Fe} / \mathrm{H}]=-0.38$ for $0.0^{\prime}<R \leq 2.5^{\prime}$, at $[\mathrm{Fe} / \mathrm{H}]=-0.45$ for $2.5^{\prime}<R \leq 5.0^{\prime}$, and at $[\mathrm{Fe} / \mathrm{H}]=-0.57$ for $5.0^{\prime}<R \leq 9.0^{\prime}$. As weak as it may appear, the change of metallicity distribution of the metal-rich $([\mathrm{Fe} / \mathrm{H}] \geq-1.0)$ component is demonstrated to be real and statistically significant in Fig. 8. The probability that the Sgr, N stars within and outside a radius of $4.0^{\prime}$ from the centre are extracted from the same parent distribution of $[\mathrm{Fe} / \mathrm{H}]$ is $P=0.004$ per cent, according to a Kolmogorov-Smirnov test, the innermost sub-sample being clearly skewed toward higher metallicities. It is unclear if the gradient on such a small scale $(\$ 100 \mathrm{pc})$ is related to the kpc-scale gradient that is seen in the main-body at large and in the Stream. Still it provides a relevant additional constraint to models for the formation of the Sgr nucleus.

In their analysis of the stellar populations in the innermost $2.5^{\prime}$ of Sgr, N, based on Advanced Camera for Surveys/Hubble Space Telescope data, Siegel et al. (2007) associated the multiple turn-offs (TO) seen in their CMD (above the old and metalpoor one dominated by M54 stars) with up to four subsequent bursts of star formation with increasing metallicity. It is tantalising to conclude that the metallicity gradient described above corresponds to an age gradient, with more recent and more metal-rich bursts occurring closer to the centre of the system, 


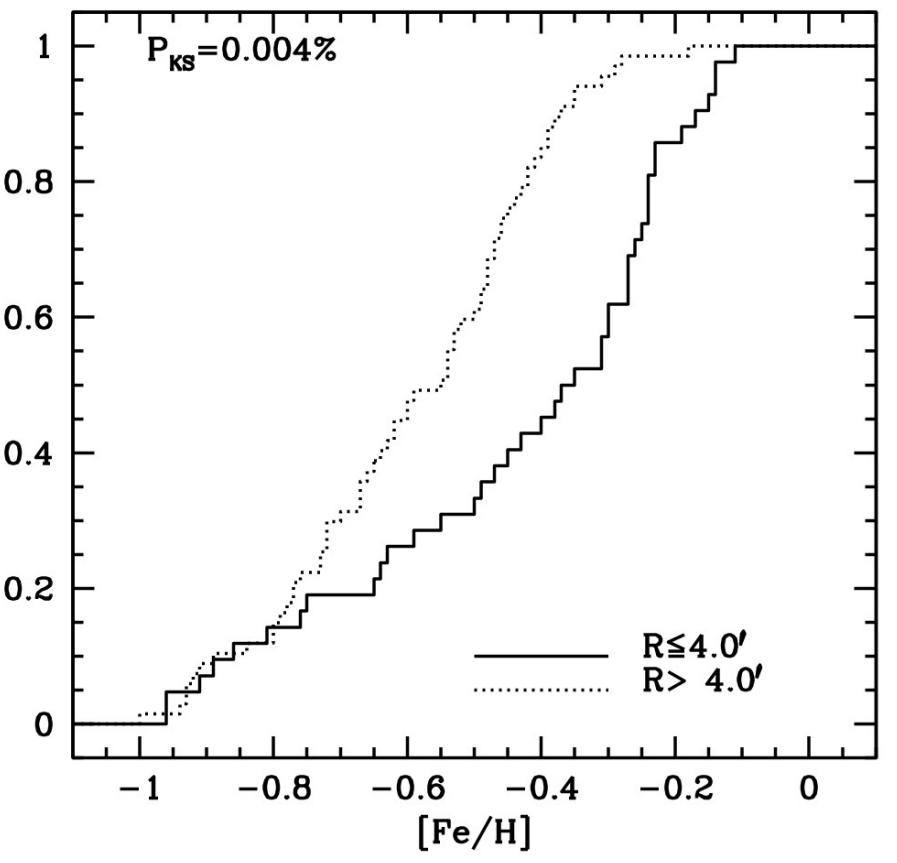

Fig. 8. Cumulative metallicity distribution for stars with $[\mathrm{Fe} / \mathrm{H}] \geq-1.0$ in two different radial ranges. The probability that the two distributions are extracted from the same parent population according to a Kolmogorov-Smirnov test $\left(P_{\mathrm{KS}}\right)$ is labelled.

that is with a smaller characteristic length scale. In particular Siegel et al. (2007) identify a population with age $=4-6 \mathrm{Gyr}$ and $[\mathrm{Fe} / \mathrm{H}] \simeq-0.6$ (possibly split into two sub populations), a population with age $=2.3 \mathrm{Gyr}$ and $[\mathrm{Fe} / \mathrm{H}] \simeq-0.1$, and a blue plume population that can be interpreted as having age $=0.1-$ $0.8 \mathrm{Gyr}$ and $[\mathrm{Fe} / \mathrm{H}] \simeq+0.6$ or as being largely composed of blue stragglers (BSS, see Ferraro 2016, and references therein). This multi-modality in mean age-metallicity is not reflected in the metallicity distribution shown in Fig. 7, but this does not seem particularly significant since multiple peaks in a relatively narrow metallicity range would be easily wiped out by the effect of observational uncertainty on individual metallicity estimates.

On the other hand, we used the Siegel et al. (2007) photometry ${ }^{6}$ (adopting similar selections on quality parameters) to compare the radial distribution of the three main sub-populations. The adopted selection strictly follows the age-metallicity classification by Siegel et al. (2007) and is illustrated in the upper panel of Fig. 9. The TO stars used to trace the various populations can be clearly recognised in the CMD; they are more than six magnitudes above the limiting magnitude of the overall photometry and cover similar ranges in magnitude, therefore they should have very similar completeness as a function of distance from the centre of the system. For this reason, their radial distributions can be safely compared, as done in the lower panel of Fig. 9. This plot shows that the age $=2.3 \mathrm{Gyr} /[\mathrm{Fe} / \mathrm{H}] \simeq$ -0.1 population is more centrally concentrated than the age $=4-6 \mathrm{Gyr} /[\mathrm{Fe} / \mathrm{H}] \simeq-0.6$, for $R \leq 2.5^{\prime}$. This implies that the more metal-rich population dominates the innermost region while the one with mean metallicity $[\mathrm{Fe} / \mathrm{H}] \simeq-0.6$ is likely to become increasingly relevant at larger radii, in excellent agreement with our findings based on abundance analysis. According to a Kolmogorov-Smirnov test, the probability that the radial distribution of the two samples is extracted from the same parent 6 Retrieved from http://www.astro.ufl.edu/ ata/public
hstgc/
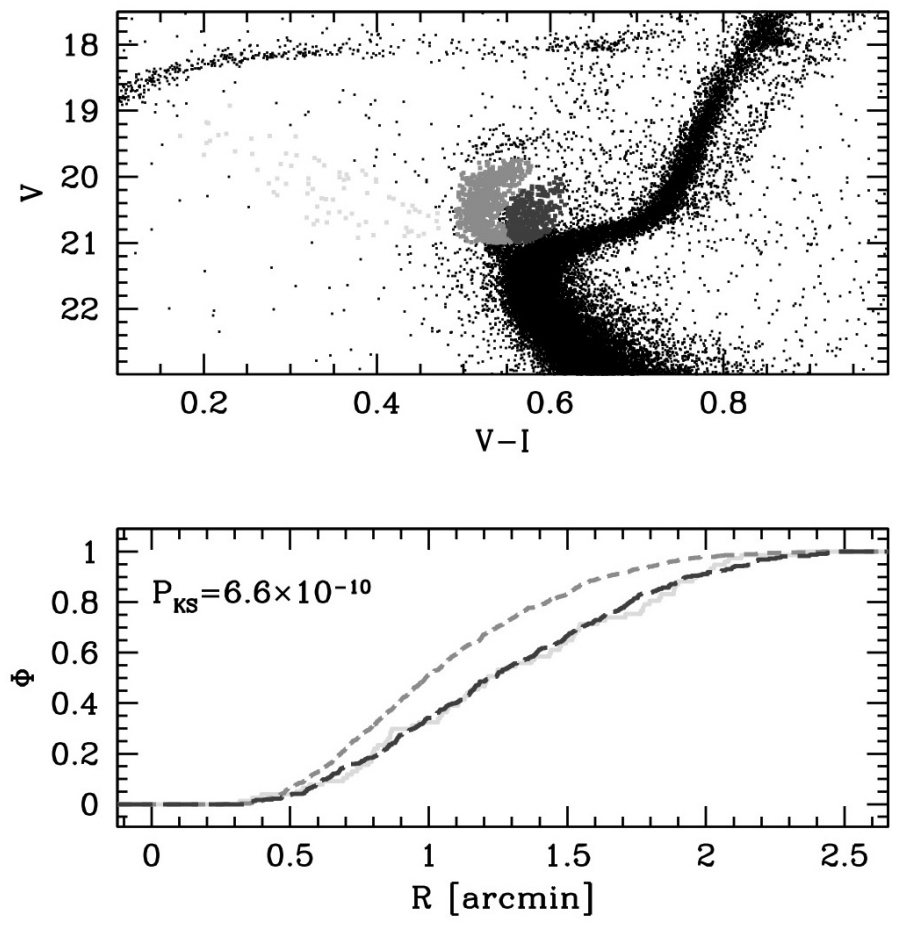

Fig. 9. Upper panel: colour magnitude diagram of the innermost $2.5^{\prime}$ from the centre of Sgr, $\mathrm{N}$ from the Advanced Camera for Surveys/Hubble Space Telescope photometry of Siegel et al. (2007). The stars associated by Siegel et al. (2007) with a possible young population (or a population of BSS), a population with age $\simeq 2.3 \mathrm{Gyr}$ and $[\mathrm{Fe} / \mathrm{H}]=-0.1$, and a population with age $\simeq 4-6 \mathrm{Gyr}$ and $[\mathrm{Fe} / \mathrm{H}]=-0.6$, are highlighted in different tones of grey, from the palest to the darkest, respectively. Lower panel: the cumulative radial distribution of the three populations are plotted using the same tones of grey (moreover, continuous line: young/BSS population; short-dashed line: 2.3 Gyr population; long-dashed line: 4-6 Gyr population). The probability that the samples tracing the $2.3 \mathrm{Gyr}$ and the 4-6 Gyr populations have the same radial distribution is reported.

distribution is $P_{\mathrm{KS}}=6.6 \times 10^{-10}$. The radial distribution of blue plume stars is indistinguishable from that of the older population, suggesting that it is most likely largely composed of BSS from both M 54 and Sgr, N. Hence both photometric and spectroscopic evidence independently suggest that the metal-rich population of Sgr, $\mathrm{N}$ formed outside-in over a timescale of a few Gyr, while the mean metallicity grew from $\simeq 1 / 4$ solar to solar.

\subsection{Trends with metallicity}

In the left-column panels of Fig. 10 we show the trends of $[\mathrm{Mg} / \mathrm{Fe}],[\mathrm{Ca} / \mathrm{Fe}]$, and $[\mathrm{Ti} / \mathrm{Fe}]$ against iron abundance for the entire sample. The comparison of the total sample with the subset of targets with uncertainties on the abundance ratio estimates lower than 0.15 dex (black points) suggests that a significant part of the spread seen at each metallicity is due to observational errors and the underlying true trends are relatively narrow in the $[\alpha / \mathrm{Fe}]$ direction.

In the right column of panels we make an attempt to minimise the contamination by M 54 stars by selecting only the handful of stars with $R \geq 6.5$, the radius out of which the surface brightness of the cluster drops below that of Sgr, according to B08 (their Fig. 2, in particular). The overall trends remain similar to those which include the full M54 population. The data available in the literature suggest that the mean $\alpha$-elements abundance of M 54 is consistent with that of $\mathrm{Sgr} \mathrm{dSph}$ as a 


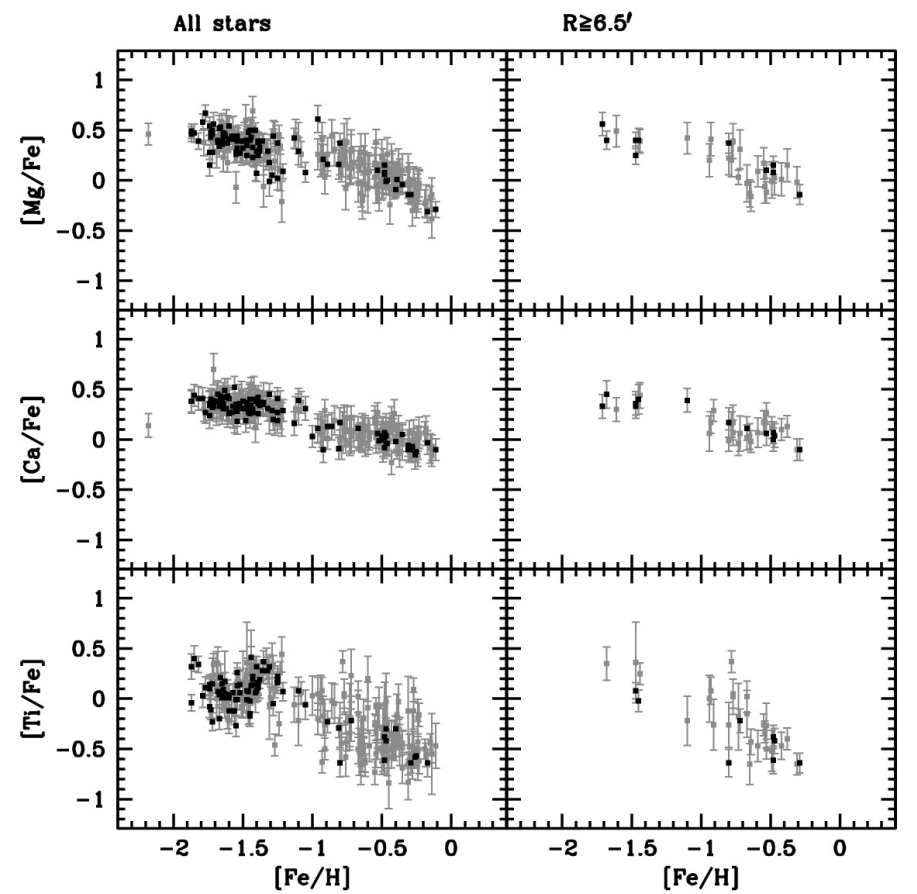

Fig. 10. Trend of all the elemental abundances considered here versus $[\mathrm{Fe} / \mathrm{H}]$ for the whole sample (left panels) and for a sub-sample minimising the contribution from M 54 stars (right panel; $R \geq 6.5^{\prime}$ ). The black dots correspond to elemental ratios whose uncertainty is $\leq 0.15$ dex.

whole in the relevant range of metallicity. The analysis of the Keck/DEIMOS spectra supports this view, consequently we will assume, as a working hypothesis, that M 54 stars are good tracers for the $\alpha$-elements abundance trends of Sgr in the metal-poor regime (but not of the radial distributions), as explained above).

Figure 11 compares the $[\alpha / \mathrm{Fe}]$ abundance ratio (defined as $0.5 \times([\mathrm{Mg} / \mathrm{Fe}]+[\mathrm{Ca} / \mathrm{Fe}]))$ as a function of $[\mathrm{Fe} / \mathrm{H}]$ of our Sgr, $\mathrm{N}$ sample with samples from different galaxies taken from the literature. The galaxies we compare with are: the Milky Way (red circles, Edvardsson et al. 1993; Burris et al. 2000; Fulbright 2000; Stephens \& Boesgaard 2002; Gratton et al. 2003; Reddy et al. 2003, 2006; Barklem et al. 2005; Bensby et al. 2005), Fornax (blue dots, Letarte et al. 2010; Lemasle et al. 2014), and Carina (cyan triangles, Lemasle et al. 2012; Fabrizio et al. 2015), in the upper panel, and Large Magellanic Cloud (LMC; green dots, Lapenna et al. 2012; Van der Swaelmen et al. 2013) in the lower panel of Fig. 11.

The metal-poor component of our sample $([\mathrm{Fe} / \mathrm{H}]<-1.0$, dominated by M 54 stars) exhibits an enhancement of the $[\alpha / \mathrm{Fe}]$ abundance ratio that matches well with those of the Galactic Halo stars and the bulk of the Carina stars. On the other hand, in the metal-rich regime the level of $\alpha$-elements abundance is intermediate between the Milky Way (on the high side) and Fornax (on the low side), at any metallicity. The inspection of the lower panel of Fig. 11 reveals that the overall pattern of $\alpha$-elements versus $[\mathrm{Fe} / \mathrm{H}]$ seen in the Sgr dSph is quite similar to that observed in the LMC, suggesting a similarity between the progenitor of Sgr dSph and the most massive of the satellites that orbit the Milky Way (see also Monaco et al. 2005b; de Boer et al. 2014; Gibbons et al. 2017).

A high-mass progenitor is hinted at also by the comparison with other dwarf spheroidal galaxies shown in Fig. 12. The $0.5 \times([\mathrm{Mg} / \mathrm{Fe}]+[\mathrm{Ca} / \mathrm{Fe}])$ versus $[\mathrm{Fe} / \mathrm{H}]$ patterns of Draco, Sculptor, and Fornax dSph's are taken from Kirby et al. (2010), who

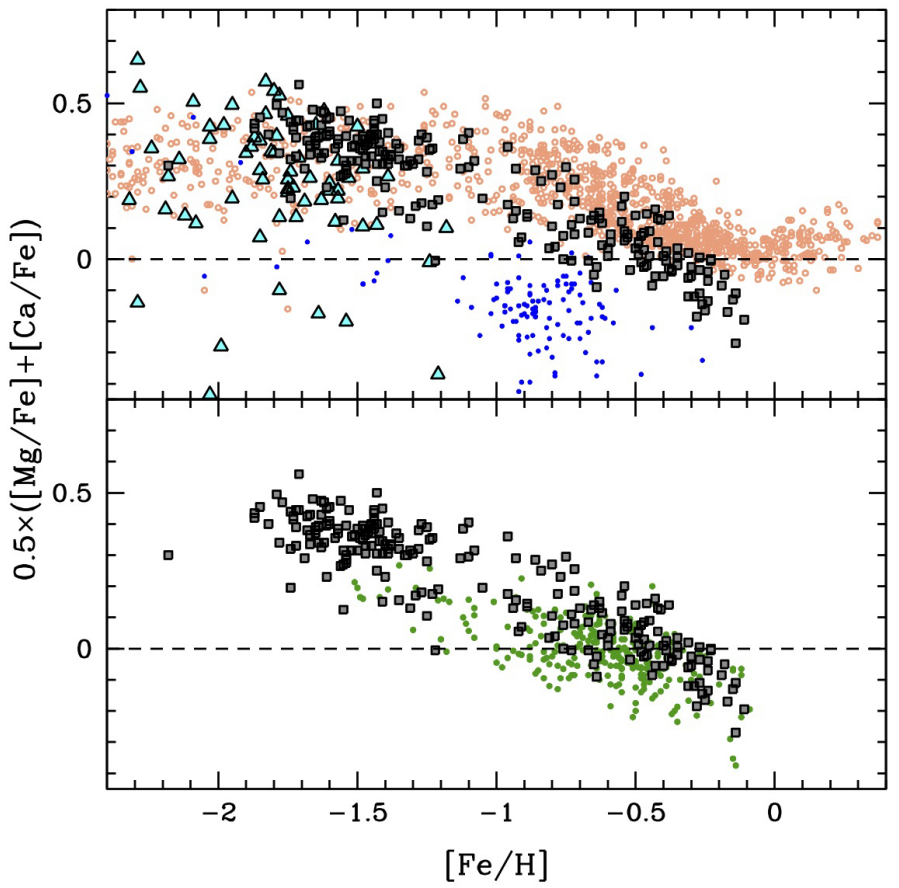

Fig. 11. Behaviour of $0.5 \times([\mathrm{Mg} / \mathrm{Fe}]+[\mathrm{Ca} / \mathrm{Fe}])$ as a function of $[\mathrm{Fe} / \mathrm{H}]$ for the whole sample (grey squares) in comparison with (upper panel) the Milky Way stars (red circles, Edvardsson et al. 1993; Burris et al. 2000; Fulbright 2000; Stephens \& Boesgaard 2002; Gratton et al. 2003; Reddy et al. 2003, 2006; Barklem et al. 2005; Bensby et al. 2005), Fornax (blue dots, Letarte et al. 2010; Lemasle et al. 2014), and Carina stars (cyan triangles, Lemasle et al. 2012; Fabrizio et al. 2015), and with (lower panel) LMC stars (green dots, Lapenna et al. 2012; Van der Swaelmen et al. 2013).

measured their abundance estimates from Keck/DEIMOS spectra with the same set-up as those analysed here. In this figure, the galaxies are ordered in the various panels according to their $V$ band absolute magnitude, a proxy for stellar mass. A trend of the maximum metallicity with $M_{V}$ is clearly evident, suggesting that the progenitor of Sgr dSph was more massive than Fornax dSph, which is the most massive among dSphs orbiting the Milky Way.

To further investigate the chemical evolution of the Sgr system and the properties of its progenitor-precursor, we recur to chemical evolution models.

\subsection{Chemical evolution of the Sagittarius complex precursor}

Chemical evolution models for Sgr in the literature refer to its bound central region (e.g. Lanfranchi \& Matteucci 2003; Vincenzo et al. 2015); in this section, we discuss a more general model for a precursor that interacts disruptively with the Galaxy leaving behind the compact body and the stellar streams we see today. It is evident that both theoretical limitations and the lack of robust observational constraints (e.g. on the total mass of the progenitor, the epoch of infall, etc.) hamper our ability to model in proper detail a system with a complex chemo-dynamical evolution like the Sgr dSph. Here we use a pure chemical model to reproduce the observed chemical abundance pattern to get broad indications on the past evolutionary path of the system.

Niederste-Ostholt et al. (2010) have re-assembled the stellar debris of the Sgr dSph and found that the stellar mass of the progenitor at infall must have been as high as $M_{\star} \simeq 6.4 \times 10^{8} M_{\odot}$ (see also Niederste-Ostholt et al. 2012), that is, more than a factor of ten higher than the present-day stellar mass of the main 


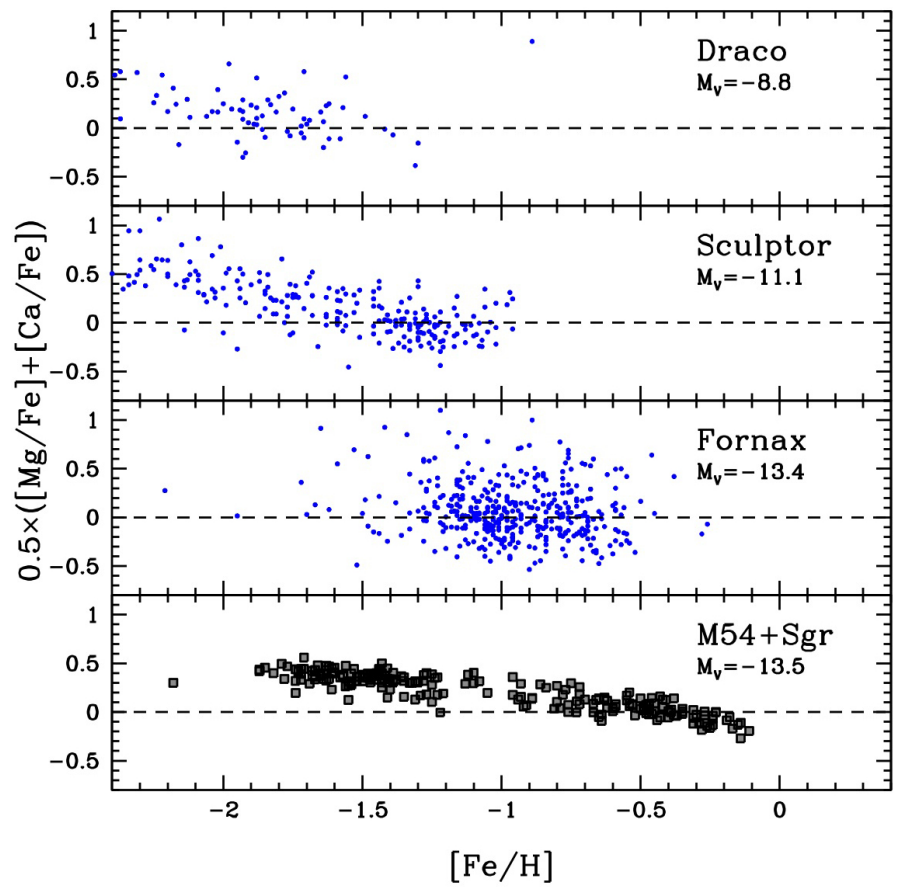

Fig. 12. Behaviour of $0.5 \times([\mathrm{Mg} / \mathrm{Fe}]+[\mathrm{Ca} / \mathrm{Fe}])$ as a function of $[\mathrm{Fe} / \mathrm{H}]$ for the whole sample (grey squares, lowest panel) in comparison with the stars of Draco, Sculptor, and Fornax dSph's analysed (blue points, Kirby et al. 2010, upper panels) using Keck/DEIMOS spectra with the same set-up as those analysed here.

body. From this, and using the stellar mass-halo mass relation from Behroozi et al. (2010), it turns out that the progenitor of Sgr was embedded in a heavy dark halo with $M_{\mathrm{DM}} \sim 10^{11} M_{\odot}$. In order to reproduce the run of velocity dispersion with longitude for the metal-poor and metal-rich populations in the Stream, Gibbons et al. (2017) set a lower limit of $6 \times 10^{10} M_{\odot}$ to the dark halo mass.

Resting on the above-mentioned observational evidence, we follow the chemical evolution of a system with $M_{\mathrm{DM}}=6 \times$ $10^{10} M_{\odot}$ and $M_{\mathrm{bar}}=0.17 M_{\mathrm{DM}}=10^{10} M_{\odot}$. A fraction of $M_{\mathrm{bar}}$ is accreted in the form of cold neutral gas according to an exponentially-decreasing law, $\mathrm{d} M_{\text {inf }} / \mathrm{d} t \propto \mathrm{e}^{-\mathrm{t} / \tau}$, where $M_{\text {inf }}=$ $2.1 \times 10^{9} M_{\odot}$ and $\tau=0.5 \mathrm{Gyr}$. This gas is turned into stars following a Kennicutt-Schmidt (Schmidt 1959; Kennicutt 1998) law, $\psi(t)=v M_{\text {gas }}(t)$, where $v=0.1-0.001 \mathrm{Gyr}^{-1}$ is the starformation efficiency and $M_{\text {gas }}(t)$ is the mass of cold neutral gas at a given time. A more detailed description of the adopted formalism can be found in Sect. 3.1 of Romano et al. (2015, and references therein).

The star-formation history of the precursor of Sgr as a whole is poorly constrained; resting on studies by Dolphin (2002) and de Boer et al. (2015), we assume that the bulk of the stars formed rather efficiently $\left(v=0.1 \mathrm{Gyr}^{-1}\right)$ from $\sim 14$ to $7 \mathrm{Gyr}$ ago, then the star-formation rate dropped abruptly $\left(v=0.001 \mathrm{Gyr}^{-1}\right)$, until the star formation stopped 6 Gyr ago. This assumption may appear in stark contrast with the results of Siegel et al. (2007). However this should be considered just as a convenient working hypothesis at this stage. We will show below that we find a good (possibly better) match with the observed chemical pattern also with models in which the abrupt stop of star formation occurs much later, while it is impossible to reproduce the observations without a significant gas-loss episode. The model assumes a Kroupa (2002) initial mass function (IMF), with a slope $x=1.7$ ( $x=1.35$ for the Salpeter IMF) in the high-mass regime. Given the relatively deep potential well in which our Sgr precursor is embedded, the internal feedback alone is unable to trigger an outflow and deprive the galaxy of its residual gas. We thus assume that, starting from 7.5 Gyr ago, because of the effects of Galactic tides, the galaxy rapidly lost its gas, together with the outermost stellar populations that form the stream. This gas stripping episode leads to the consumption of all the available gas 6 Gyr ago, in the model. Summarising the sequence of events, for clarity: (a) the model forms stars with $v=0.1 \mathrm{Gyr}^{-1}$ starting from 14 Gyr ago; (b) starting from 7.5 Gyr ago gas is lost at a rate tuned to reproduce the observed abundance patterns; (c) this gas-loss process leads to the total consumption of the gas reservoir by $6 \mathrm{Gyr}$ ago, when, consequently, the star formation ceases.

The stellar yields are from Karakas (2010) for low- and intermediate-mass stars, Nomoto et al. (2013) for massive stars, and Iwamoto et al. (1999) for type Ia supernovae. The adopted stellar nucleosynthesis from massive stars is slightly updated with respect to our previous work, so we checked that with our choice of the stellar yields the $[\mathrm{X} / \mathrm{Fe}]$ versus $[\mathrm{Fe} / \mathrm{H}]$ trends of elements from $\mathrm{C}$ to $\mathrm{Zn}$ in the Milky Way are reproduced. We run a model in which all massive stars explode as normal type II supernovae and a model in which all stars above $20 M_{\odot}$ explode as hypernovae (solid and dotted lines, respectively, in Fig. 13).

The values of the free parameters $M_{\text {inf }}, \tau$, and $v$ are fixed in such a way that the chemical properties of Sgr stars are reproduced, while a stellar mass of $M_{\star} \simeq 7 \times 10^{8} M_{\odot}$ at infall is obtained. For the comparison with the model predictions, we use data from this work for $[\mathrm{Mg} / \mathrm{Fe}]$ and $[\mathrm{Ca} / \mathrm{Fe}]$. We define theoretical $[\alpha / \mathrm{Fe}]$ ratios as $0.5 \times[\mathrm{Mg} / \mathrm{Fe}]+0.5 \times[\mathrm{Ca} / \mathrm{Fe}]$ for a twofold reason: first, we want to compare with the data presented in this work and, second, current yields of Ti still lead to theoretical $[\mathrm{Ti} / \mathrm{Fe}]$ versus $[\mathrm{Fe} / \mathrm{H}]$ trends that do not reproduce the one observed in the Milky Way (Romano et al. 2010; Kirby et al. 2011; Nomoto et al. 2013), thus making any comparison involving Ti not meaningful. For the iron-peak elements $\mathrm{Mn}, \mathrm{Cu}$, and $\mathrm{Zn}$, we use data from Carretta et al. (2010b), Sbordone et al. (2007), and McWilliam et al. (2013). Measurements of $\mathrm{Na}$ and $\mathrm{Al}$ are also available for Sgr main-body stars. However, for stars belonging to M54 these may reflect the peculiar enrichment processes occurring in a globular cluster, rather than the global galactic enrichment process. Therefore, we do not consider these elements in our study. On the other hand, the $[\mathrm{Mg} / \mathrm{Fe}]$ spread in M 54 is moderate $(\mathrm{C} 10 \mathrm{~b})$, thus it is not expected to seriously affect the general trend with $[\mathrm{Fe} / \mathrm{H}]$. The analysis of $\mathrm{Eu}$ data, instead, is complicated by the fact that this element, like other r-process elements, is likely to originate, at least partly, from compact binary mergers (Matteucci et al. 2014). Since we do not have an estimate of the rate of compact binary mergers in the progenitor of Sgr, we prefer not to deal with Eu in this paper.

From Fig. 13 it can be seen that our pure chemical evolution model, notwithstanding the rough assumptions it makes about the effects of the interaction with the Galaxy, is quite successful in reproducing the average trends of the analysed elements in Sgr. We stress that the abruptly decreasing trends of $[\mathrm{Mg} / \mathrm{Fe}]$ and $[\mathrm{Ca} / \mathrm{Fe}]$ for $[\mathrm{Fe} / \mathrm{H}]>-0.4$ (red portions of the curves in Fig. 13) can be obtained only by assuming that an efficient stripping is removing all the gas starting from $7.5 \mathrm{Gyr}$ ago; without it, a much milder decrease would be found, at variance with the observations. Comparing the predictions of a model for the whole Sgr system with data from the central nucleus alone may not appear fully appropriate, but we verified that our model reproduces satisfactorily also the $[\alpha / \mathrm{Fe}]$ versus $[\mathrm{Fe} / \mathrm{H}]$ pattern found 

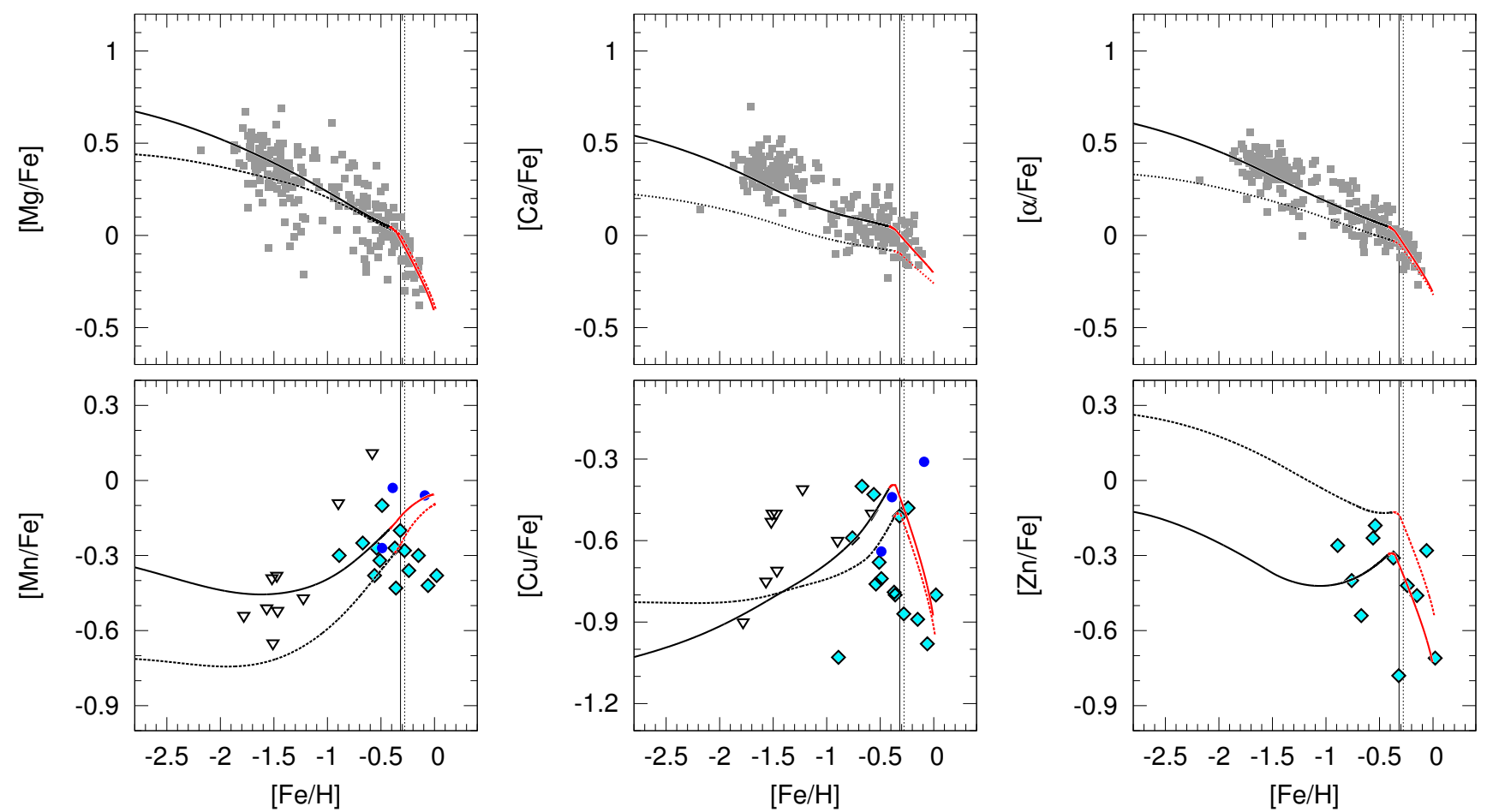

Fig. 13. Run of $[\mathrm{X} / \mathrm{Fe}]$ versus $[\mathrm{Fe} / \mathrm{H}]$ for - clockwise from top left - $\mathrm{Mg}, \mathrm{Ca}, \alpha$-elements, $\mathrm{Zn}, \mathrm{Cu}$, and $\mathrm{Mn}$ predicted with our chemical evolution model assuming that stars above $20 M_{\odot}$ explode as normal type II supernovae (solid curves) or as hypernovae (dotted curves). The red portions of the curves show the phases in which the stripping is active, while the vertical lines mark the end points of the tracks without stripping. The theoretical predictions are compared to data from this work (grey filled squares), Sbordone et al. (2007, cyan diamonds), Carretta et al. (2010b, upside-down triangles), and McWilliam et al. (2013, blue filled circles).

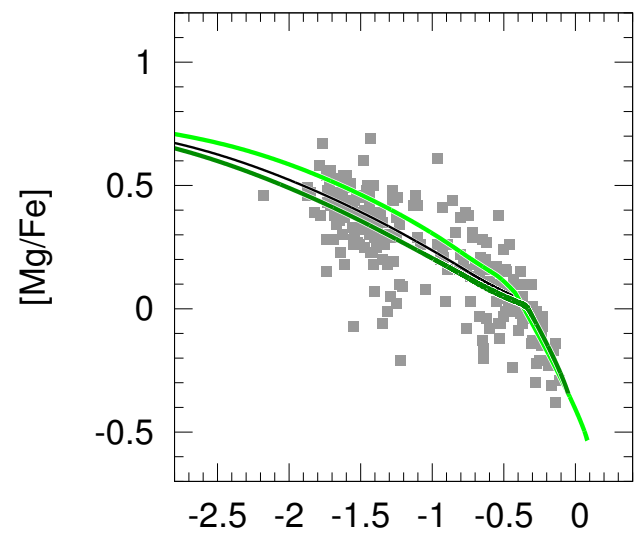

$[\mathrm{Fe} / \mathrm{H}]$

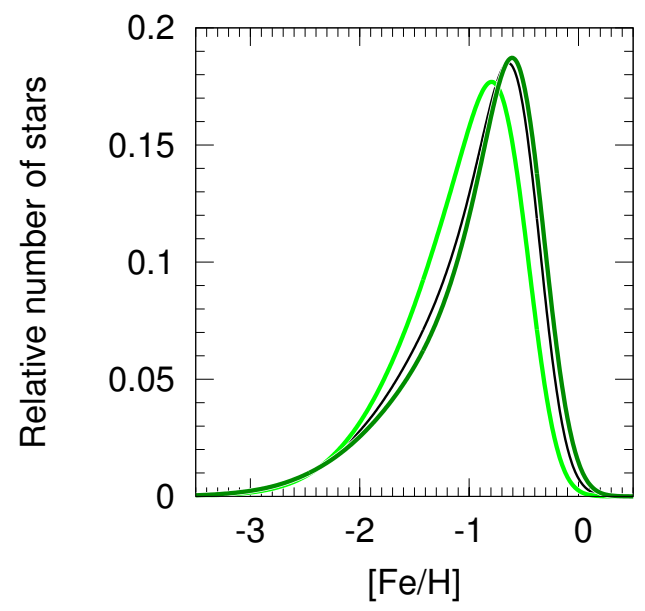

Fig. 14. Effect of a prolonged (dark green curves, gas stipping starting $3.5 \mathrm{Gyr}$ ago) or shortened (light green curves, gas stripping starting $11.5 \mathrm{Gyr}$ ago) star-formation activity on the predicted $[\mathrm{Mg} / \mathrm{Fe}]$ versus $[\mathrm{Fe} / \mathrm{H}]$ behaviour (left-hand panel) and stellar metallicity distribution (right-hand panel) of Sagittarius stars.

by de Boer et al. (2014) in the Sgr Stream ${ }^{7}$, in the metallicity range spanned by their data.

The model with normal supernovae is favoured, at least for $[\mathrm{Fe} / \mathrm{H}]>-2.0$, while the only very metal-poor stars seem to suggest that hypernovae may have played a role at early epochs. It would be interesting to test this hypothesis by measuring the abundance ratios in metal-poor stars for other elements, such as $\mathrm{Zn}$ or $\mathrm{Mn}$, whose nucleosynthesis is strongly affected by the

\footnotetext{
7 We note that de Boer et al. (2014) adopt another definition for $[\alpha / \mathrm{Fe}]$, $[\alpha / \mathrm{Fe}]=0.5 \times[\mathrm{Mg} / \mathrm{Fe}]+0.3 \times[\mathrm{Ti} / \mathrm{Fe}]+0.1 \times[\mathrm{Ca} / \mathrm{Fe}]+0.1 \times[\mathrm{Si} / \mathrm{Fe}]$. This is the reason why we do not include their data in Fig. 13.
}

explosion energy. Furthermore, it is reasonable to assume that a multi-zone model would be better suited to describe the evolution of Sgr precursors, since the outer parts of the galaxy are expected to evolve more slowly than the inner parts. Indeed, de Boer et al. (2014) find that the knee in the $[\alpha / \mathrm{Fe}]$ versus $[\mathrm{Fe} / \mathrm{H}]$ relation for stream stars occurs at $[\mathrm{Fe} / \mathrm{H}] \sim-1.2 \mathrm{dex}$, while our main-body sample points to a higher metallicity value of $[\mathrm{Fe} / \mathrm{H}] \sim-0.4$ dex. Much more detailed (and homogeneous) chemical abundance data for a stream sample as large as that presented in this work are required before we can attempt this kind of modelling. Also, we remark that the constraints on the 
timescales of chemical enrichment and stripping in Sgr that can be set with arguments based on the trends of abundance ratios with metallicity alone are quite loose: by assuming a shortlasting, more efficient main episode of star formation from 14 to 10.5 Gyr ago, with stripping starting $11 \mathrm{Gyr}$ ago, or a longlasting (star formation from 14 to $1 \mathrm{Gyr}$ ago, with a drop in efficiency $2 \mathrm{Gyr} \mathrm{ago}^{8}$ ), less efficient main episode of star formation and stripping starting 3.5 Gyr ago, we obtain almost the same $[\mathrm{Mg} / \mathrm{Fe}]$ versus $[\mathrm{Fe} / \mathrm{H}]$ theoretical trend (Fig. 14, left panel). The stellar metallicity distribution function, instead, could be a better diagnostic for the epoch of gas-stripping, since its peak moves from $[\mathrm{Fe} / \mathrm{H}]=-0.85$ to $[\mathrm{Fe} / \mathrm{H}]=-0.6$ in the two extreme cases considered here. One would need accurate $[\mathrm{Fe} / \mathrm{H}]$ measurements for a complete sample of Sgr stars to be able to discriminate among different scenarios from the metallicity distribution; however, models with later gas-stripping produce distributions peaking at higher metallicity, in better agreement with the observed peak of the metal-rich component of Sgr. Finally, we note that our model assumes a Kroupa (2002) IMF with a slope $x=1.7$, that is, slightly steeper than Salpeter, as usually adopted in models of the solar neighbourhood region. All in all, it seems that a more exotic IMF strongly suppressing the high-mass stars, as proposed by MWM13, is not required in order to explain the available data.

\section{Summary and conclusions}

The main results obtained from the analysis of 235 giant stars in the globular cluster M 54 and the nuclear region of the Sagittarius dwarf galaxy, Sgr, N, are summarised as follows:

- We detect for the first time a metallicity gradient within the nucleus Sgr, N. The peak of the metal-rich component shifts from $[\mathrm{Fe} / \mathrm{H}]=-0.38$ for $R \leq 19.0 \mathrm{pc}$ to $[\mathrm{Fe} / \mathrm{H}]=-0.57$ for $38.0 \mathrm{pc}<R \leq 70.0 \mathrm{pc}$ (projected distance). This evidence, together with the radial distribution of stars of different ages and metallicities in the innermost $\simeq 20 \mathrm{pc}$, indicates that the stars in the metal-rich component of Sgr, $\mathrm{N}$ formed outsidein over a few Gyr, with each subsequent generation of stars more centrally concentrated than the previous one.

- Hence, the Sgr, N stellar population is not dynamically mixed and retains, in this very confined spatial region, some memory of its formation. We showed previously in B08 that the timescale for M54 to decay into the centre of Sgr by dynamical friction is very short, on the order of approximately a Gyr. During this process, the globular cluster would have caused a massive wake in the ambient medium, which would have stirred the inner regions very effectively. It is unlikely that any pre-existing structure such as Sgr, $\mathrm{N}$ could have survived as a chemically inhomogeneous structure after this mixing. This opens up the fascinating possibility of being able to place an upper limit to the time since the arrival of M 54 into the Sagittarius core by measuring the age of the Sgr, N stellar population. In the framework outlined by Siegel et al. (2007), this would suggest that the infall of M 54 occurred more than $~ 6 \mathrm{Gyr}$ ago. Indeed, it is possible that this massive and compact system may have had a role in collecting the enriched gas that went on to form Sgr, $\mathrm{N}$ at the very centre of the Sagittarius galaxy.

- Assuming that M54 shares the same chemical pattern as Sgr dSph stars in the metallicity range spanned by its stars,

\footnotetext{
8 We note that this model is broadly consistent with the star-formation scenario emerging from the analysis by Siegel et al. (2007).
}

we recover the $[\mathrm{Mg} / \mathrm{Fe}],[\mathrm{Ca} / \mathrm{Fe}]$, and $[\mathrm{Ti} / \mathrm{Fe}]$ versus $[\mathrm{Fe} / \mathrm{H}]$ trend at the centew of $\mathrm{Sgr}$ from $[\mathrm{Fe} / \mathrm{H}] \simeq-2.2$ to $[\mathrm{Fe} / \mathrm{H}] \simeq$ -0.2 .

- For $[\mathrm{Fe} / \mathrm{H}]<-1.0$ the $\alpha$-element abundance ratio in Sgr is the same as in the halo of the Milky Way and in classical $\mathrm{dSphs}$. On the other hand, at higher metallicity it is different from galaxy to galaxy: Sgr being intermediate between the Milky Way and Fornax dSph.

- In the $0.5 \times([\mathrm{Mg} / \mathrm{Fe}]+[\mathrm{Ca} / \mathrm{Fe}])$ versus $[\mathrm{Fe} / \mathrm{H}]$ diagram Sgr stars follow a trend very similar to LMC stars. This may suggest a similarity (e.g. in mass) between the progenitor of Sgr and the LMC, in good agreement with recent estimates of the Sgr mass at infall (Niederste-Ostholt et al. 2012; de Boer et al. 2014; Gibbons et al. 2017). The comparison in the same plane with other dSphs corroborates this conclusion.

- We reproduce the main observed abundance trends of Sgr dSph with a chemical evolution model implying an episode of strong gas loss occurring from 7.5 Gyr to $2.5 \mathrm{Gyr}$ ago, presumably starting at the first peri-Galactic passage of the dwarf after its infall into the Milky Way. In our model the gas stripping event is required to reproduce the observed abundance trends in the most metal-rich regime $([\mathrm{Fe} / \mathrm{H}] \gtrsim$ $-0.5)$. However, because of the lack of an observed stellar metallicity distribution function for a complete sample of Sgr stars, the timescales for the occurrence of the stripping and chemical enrichment can be only poorly-constrained and the one presented here is only one among different possible evolutive scenarios.

Acknowledgements. We are grateful to the anonymous referee for the useful comments and suggestions. D.R. benefited from the International Space Science Institute (ISSI, Bern, $\mathrm{CH}$ ), thanks to the funding of the team "The Formation and Evolution of the Galactic Halo". Most of the data presented herein were obtained at the W. M. Keck Observatory, which is operated as a scientific partnership between the California Institute of Technology, the University of California, and the National Aeronautics and Space Administration. The Observatory was made possible by the generous financial support of the W. M. Keck Foundation, partially based on Advanced Camera for Surveys observations collected with the HST within the programme GO-1077. This research has made use of the SIMBAD database, operated at CDS, Strasbourg, France. This research has made use of the NASA/IPAC Extragalactic Database (NED) which is operated by the Jet Propulsion Laboratory, California Institute of Technology, under contract with the National Aeronautics and Space Administration. This research has made use of NASA's Astrophysics Data System.

\section{References}

\section{Alard, C. 2001, A\&A, 377, 389}

Alonso, A., Arribas, S., \& Martinez-Roger, C. 1999, A\&AS, 140, 261 Barklem P. S., Christlieb, N., Beers, T. C., et al. 2005, A\&A, 439, 129 Behroozi, P. S., Conroy, C., \& Wechsler, R. H. 2010, ApJ, 717, 379 Bellazzini, M., Ferraro, F. R., \& Buonanno, R. 1999, MNRAS, 307, 619 Bellazzini, M., Ferraro, F. R., \& Ibata, R.A. 2003, AJ, 125, 188

Bellazzini, M., Newberg, H. J., Correnti, M., Ferraro, F. R., \& Monaco, L. 2006a, A\&A, 457, L21

Bellazzini, M., Correnti, M., Ferraro, F. R., Monaco, L.,\& Montegriffo, P. 2006b, A\&A, 446, L1

Bellazzini, M., Ibata, R. A., Chapman, S. C., et al. 2008, AJ, 136, 1147 (B08) Bellazzini, M., Beccari, G., Oosterloo, T. A., et al. 2011, A\&A, 527, A58 Belokurov, V., Koposov, S. E., Evans, N. W., et al. 2014, MNRAS, 437, 116 Bensby, T., Feltzing, S., Lundstrom, I., \& Ilyin, I. 2005, A\&A, 433, 185 Bessell, M. S. 1979, PASP, 91, 589

Bonifacio, P., Hill, V., Molaro, P., et al. 2000, A\&A, 359, 663

Bonifacio, P., Sbordone, L., Marconi, G., Pasquini, L., \& Hill, V. 2004, A\&A, 414, 503

Burris, D. L., Pilachowski, C. A., Armandroff, T. E., et al. 2000, ApJ, 544, 302

Carrera, R., Gallart, C., Pancino, E., \& Zinn, R. 2007, AJ, 134, 1298

Carretta, E., Bragaglia, A., Gratton, R. G., et al. 2009, A\&A, 505, 117

Carretta, E., Bragaglia, A., Gratton, R. G., et al. 2010a, ApJ, 714, L7 (C10a) 
Carretta, E., Bragaglia, A., Gratton, R. G., et al. 2010b, A\&A, 520, A95 (C10b) de Boer, T. J. L., Belokurov, V., Beers, T. C., \& Lee, Y. S. 2014, MNRAS, 443 , 658

de Boer, T. J. L., Belokurov, V., \& Koposov, S. 2015, MNRAS, 451, 3489

Chou, M.-Y., Majewski, S. R., Cunha, K., et al. 2007, ApJ, 670, 346

Dolphin, A. E. 2002, MNRAS, 332, 91

Edvardsson, B., Andersen, J., Gustafsson, B., et al. 1993, A\&A, 275, 101

Fabrizio, M., Nonino, M., Bono, G., et al. 2015, A\&A, 580, A18

Ferraro, F. R. 2016, in Star Clusters and Black Holes in Galaxies across Cosmic Time, IAU Symp. 312, 171

Fulbright, J. P. 2000, AJ, 120, 1841

Gibbons, S. L. J., Belokurov, V., \& Evans, N. W. 2017, MNRAS, 464, 794

Gratton, R. G., Carretta, E., Claudi, R., Lucatello, S., \& Barbieri, M. 2003, A\&A 404, 187

Grcevich, J., \& Putman, M. E. 2009, ApJ, 696, 385

Hamanowicz, A., Pietrukowicz, P., Udalski, A., et al. 2016, A\&A, 66, A197

Hyde, E. A., Keller, S., \& Zucker, D. B. 2015, ApJ, 805, 189

Ibata, R. A., Gilmore, G., \& Irwin, M. J. 1994, Nature, 370, 194

Ibata, R., Irwin, M., Lewis, G. F., \& Stolte, A. 2001, ApJ, 547, 133

Ibata, R, Chapman, S., Ferguson, A. M. N., et al. 2004, MNRAS, 351, 1171

Ibata, R., Bellazzini, M., Chapman, S.C., et al. 2009, ApJ, 699, L169

Iwamoto, K., Brachwitz, F., Nomoto, K., et al. 1999, ApJS, 125, 439

Karakas, A. I. 2010, MNRAS, 403, 1413

Keller, S. C., Yong, D., \& Da Costa, G. S. 2010, ApJ, 720, 940

Kennicutt, R. C., Jr. 1998, ApJ, 498, 541

King, I. 1962, AJ, 67, 471

Kirby, E. N., Guhathakurta, P., Bolte, M., Sneden, C., \& Geha, M. 2009, ApJ, 705,328

Kirby, E. N., Guhathakurta, P., Simon, J. D., et al. 2010, ApJS, 191, 352

Kirby, E. N., Cohen, J. G., Smith, G. H., et al. 2011, ApJ, 727, 79

Kroupa, P. 2002, ASP Conf. Ser., 285, 86

Lanfranchi, G. A., \& Matteucci, F. 2003, MNRAS, 345, 71

Lapenna, E., Mucciarelli, A., Origlia, L., \& Ferraro, F. R. 2012, ApJ, 761, 33

Layden, A. C., \& Sarajedini, A. 2000, AJ, 119, L1760

Law, D. R., \& Majewski, S. R. 2010, ApJ, 714, 229

Letarte, B., Hill, V., Tolstoy, E., et al. 2010, A\&A, 523, A17

Lemasle, B., Hill, V., Tolstoy, E., et al. 2012, A\&A, 538, A100

Lemasle, B., de Boer, T. J. L., Hill, V., et al. 2014, A\&A, 572, A88
Majewski, S. R., Skrutskie, M. F., Weinberg, M. D., \& Ostheimer, J.C. 2003, ApJ, 599, 1082

Majewski, S. R., Hasselquist, S., Lokas, E. L., et al. 2013, ApJ, 777, L13 Matteucci, F., Romano, D., Arcones, A., et al. 2014, MNRAS, 438, 2177 McConnachie, A. W. 2012, AJ, 144, 4

McWilliam, A., Wallerstein, G., \& Mottini, M. 2013, ApJ, 778, 149 (MWM13) Monaco, L., Ferraro, F. R., Bellazzini, M., \& Pancino, E. 2002, ApJ, 578, L47

Monaco, L., Bellazzini, M., Ferraro, F. R., \& Pancino, E. 2003, ApJ, 597, L25

Monaco, L., Bellazzini, M., Ferraro, F. R., \& Pancino, E. 2004, MNRAS, 349, 1278

Monaco, L., Bellazzini, M., Ferraro, F. R., \& Pancino, E. 2005a, MNRAS, 356, 1396

Monaco, L., Bellazzini, M., Bonifacio, P., et al. 2005b, A\&A, 441, 141 (M05b)

Monaco, L., Bellazzini, M., Bonifacio, P., et al. 2007, A\&A, 464, 201

Mucciarelli, A., Bellazzini, M., Ibata, R., et al. 2012, MNRAS, 426, 2889

Niederste-Ostholt, M., Belokurov, V., Evans, N. W., \& Peñarrubia, J. 2010, ApJ, 712,516

Niederste-Ostholt, M., Belokurov, V., \& Evans, N. W. 2012, MNRAS, 422, 207

Nomoto, K., Kobayashi, C., \& Tominaga, N. 2013, ARA\&A, 51, 457

Reddy, B. E., Tomkin, J., Lambert, D. L., \& Allende Prieto, C. 2003, MNRAS, 340,304

Reddy, B. E., Lambert, D. L., \& Allende Prieto, C. 2006, MNRAS, 367, 1329

Romano, D., Karakas, A. I., Tosi, M., \& Matteucci, F. 2010, A\&A, 522, A32

Romano, D., Bellazzini, M., Starkenburg, E., \& Leaman, R. 2015, MNRAS, 446, 4220

Sarajedini, A., \& Layden, A. C. 1995, AJ, 109, 1086

Sbordone, L., Bonifacio, P., Buonanno, R., et al. 2007, A\&A, 465, 815 (S07)

Sbordone, L., Monaco, L., Moni Bidin, C., et al. 2015, A\&A, 579, A104

Schlegel, D., Finkbeiner, D., \& Davis, M. 1998, ApJ, 500, 525

Schmidt, M. 1959, ApJ, 129, 243

Shetrone, M. D., Siegel, M. H., Cook, D. O., \& Bosler, T. 2009, AJ, 137, 62

Skrutskie, M. F., Cutri, R. M., Stiening, R., et al. 2006, AJ, 131, 1163

Siegel, M. H., Dotter, A., Majewski, S.R., et al. 2007, ApJ, 667, L57

Smecker-Hane, T. A., \& McWilliam, A. 2002, [arXiv: astro-ph/0205411]

Stephens, A., \& Boesgaard, A. M. 2002, AJ, 123, 1647

van der Swaelmen, M., Hill, V., Primas, F., \& Cole, A. A. 2013, A\&A, 560, A44

Vincenzo, F., Matteucci, F., Recchi, S., et al. 2015, MNRAS, 449, 1327 